\title{
The stellar content of the XMM-Newton bright serendipitous survey ${ }^{\star} \star \star$
}

\author{
J. López-Santiago ${ }^{1}$, G. Micela ${ }^{1}$, S. Sciortino ${ }^{1}$, F. Favata ${ }^{2}$, A. Caccianiga ${ }^{3}$, R. Della Ceca ${ }^{3}$, \\ P. Severgnini ${ }^{3}$, and V. Braito ${ }^{4,5}$
}

1 INAF - Osservatorio Astronomico di Palermo Giuseppe S. Vaiana, Piazza Parlamento 1, 90134 Palermo, Italy e-mail: jlopez@astropa.unipa.it

2 Astrophysics Missions Division, Research and Science Support Department of ESA/ESTEC, Postbus 299, 2200 AG Noordwijk, The Netherlands

3 INAF - Osservatorio Astronomico di Brera, via Brera 28, 20121 Milan, Italy

${ }^{4}$ X-ray Astrophysics Laboratory, Code 662, NASA/Goodard Space Flight Center, Greenbelt, MD 20771, USA

5 Department of Physics and Astronomy, Johns Hopkins University, Baltimore, MD 21218, USA

Received 26 May 2006 / Accepted 2 October 2006

\section{ABSTRACT}

\begin{abstract}
Context. The comparison of observed counts in a given sky direction with predictions by Galactic models yields constraints on the spatial distribution and the stellar birthrate of young stellar populations. The XMM-Newton Bright Serendipitous Survey (XBSS) is an unbiased survey that includes a total of 58 stellar sources selected in the $0.5-4.5 \mathrm{keV}$ energy band, having a limiting sensitivity of $10^{-2} \mathrm{cnt} \mathrm{s}^{-1}$ and covering an area of $28.10 \mathrm{sq} \mathrm{deg}$.

Aims. We present the results of analysing the stellar content of the XBSS so as to understand the recent star formation history of the Galaxy in the vicinity of the Sun.

Methods. We compared the observations with the predictions obtained with XCOUNT, a model of the stellar X-ray content of the Galaxy. The model predicts the number and properties of the stars to be observed in terms of magnitude, colour, population and $f_{\mathrm{x}} / f_{\mathrm{v}}$ ratio distributions of the coronal sources detected with a given instrument and sensitivity in a specific sky direction.

Results. As in other shallow surveys, we observe an excess of stars not predicted by our Galaxy model. Comparing the colours of the identified infrared counterparts with the model predictions, we observe that this excess is produced by yellow $(\mathrm{G}+\mathrm{K})$ stars. The study of the X-ray spectrum of each source reveals a main population of stars with the coronal temperature stratification typical of intermediate-age stars. As no assumptions have been made for the selection of the sample, our results must be representative of the entire solar neighbourhood. Some stars show infrared excess due to circumstellar absorption, which is indicative of youth.
\end{abstract}

Key words. Galaxy: stellar content - stars: activity - stars: coronae - stars: formation - stars: magnetic fields - X-rays: stars

\section{Introduction}

It is well known from the study of young open clusters that magnetic activity traced by coronal X-ray emission evolves during the star's life. The average $L_{\mathrm{x}}$ decreases from $\sim 10^{30} \mathrm{erg} \mathrm{s}^{-1}$ to $\sim 10^{27} \mathrm{erg} \mathrm{s}^{-1}$ during the life of normal late-type stars (Micela et al. 1985, 1988; Guedel et al. 1997; Feigelson \& Montmerle 1999). This decrease mostly occurs between the zero-age main sequence (ZAMS) and the solar age, while it is nearly negligible during the pre-main sequence (PMS) phase. As a consequence, $\mathrm{X}$-ray flux-limited surveys detect young (PMS and ZAMS) stars up to larger distances than old (main sequence, MS) ones. As a result, shallow stellar X-ray-selected samples will be dominated by young sources, while old stars will be the dominant population in deep high-latitude stellar X-ray samples, since young

\footnotetext{
* The XMM-Newton Bright Serendipitous Survey was conceived by the XMM-Newton Survey Science Center (SSC), a consortium of 10 institutions appointed by ESA to help the SOC in distinct technical aspects, including the exploitation of the XMM-Newton serendipitous detections (see http://xmmssc-www.star.le.ac.uk/).

$\star \star$ Tables 2 and 3 are only available in electronic form at http://www . aanda.org
}

sources have lower scale heights than the limiting distance of the survey (see Micela 2003, and references therein).

Stellar X-ray surveys play an important role in understanding the recent star formation history in our Galaxy in the past billion years (e.g. Favata et al. 1992). The comparison between $\mathrm{X}$-ray observations and predictions derived from X-ray galactic models can constrain the properties of stellar populations, such as their spatial distributions and the stellar birthrate in the solar neighbourhood. Thus, X-ray surveys are a very useful tool for studying the stellar population in the (near) Galaxy.

A computational model for the prediction and analysis of the distribution of galactic X-ray stellar coronal sources counts, XCOUNT, was developed by Favata et al. (1992). The model allows the prediction of the number and properties, in terms of magnitude, colour, population, and $f_{\mathrm{x}} / f_{\mathrm{v}}$ ratio distributions of the stellar coronal sources detected with a given instrument and sensitivity in a specific sky direction.

One of the first results of the comparison between predictions and observations of the Extended Medium Sensitivity Survey of Einstein (EMSS, Gioia et al. 1990) was the detection of an excess of yellow stars in the observations (Favata et al. 1988), later confirmed to be due to young (Pleiades-age) stars (Favata et al. 1993; Sciortino et al. 1995). An excess of young 


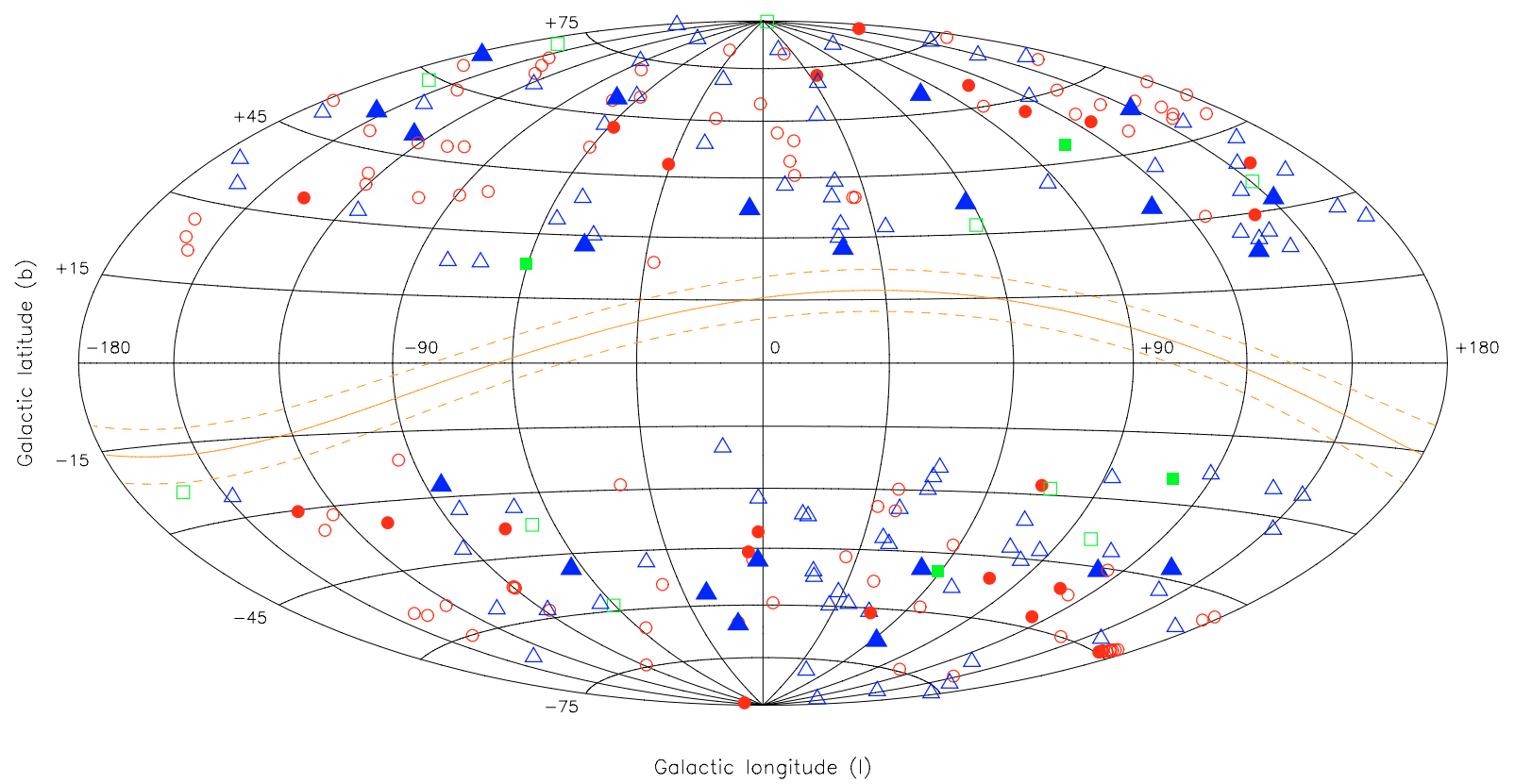

Fig. 1. Galactic distribution of the fields of the XBSS (Della Ceca et al. 2004): circles, triangles, and squares are used to represent observations carried out using the Thin, Medium, and Thick filters in MOS2, respectively. Filled symbols are plotted for those fields where stellar counterparts have been identified. The position of the Gould Belt is shown (continuous line), with a width of $\pm 5^{\circ}$ (dashed lines).

stars in the solar neighbourhood was also detected by Exosat and Rosat (e.g. Tagliaferri et al. 1994). These results exclude scenarios with decreasing stellar birthrate in the past billion years, although they are unable to distinguish between a constant and an increasing stellar birthrate hypotheses, because of the limited sample size (Micela et al. 1993). In contrast, the analysis of the Chandra Deep Field-North (CDF-N, Brandt et al. 2001) shows a lack of $\mathrm{F}, \mathrm{G}$, and $\mathrm{K}$ dwarfs compared to the predictions (Feigelson et al. 2004). The number of dM stars agrees very well with what is predicted by the model, but there is an excess of $\mathrm{dF}$, $\mathrm{dG}$, and dK stars in the predictions (Micela 2003; Feigelson et al. 2004). The observed lack of yellow stars is in the opposite direction of the discrepancy obtained with the EMSS, although it must be noticed that the CDF-N is dominated by old-disk stars, while young (PMS and ZAMS) stars are dominant in the EMSS. In the same way, a first inspection of the medium-deep X-ray survey HELLAS2XMM (Baldi et al. 2002) shows an excess of predicted stars (Micela et al. 2003). Old ( $>1$ Gyr) stars dominate with the intermediate-age $(0.1-1 \mathrm{Gyr})$ stars contributing no more than $23 \%$, while the contribution of very young $(0.01-0.1 \mathrm{Gyr})$ stars is negligible.

The discrepancy between the results of shallow and mediumdeep surveys could be explained by a non-constant stellar birthrate and/or incorrect assumptions on the spatial distribution of stars and its dependency on age in the galactic model. On one hand, an increased star formation rate in the past billion years would account for the excess of young stars in shallow surveys. On the other hand, a density distribution decreasing with distance from the Galactic plane much more steeply than the assumed exponential shape (e.g. Guillout et al. 1996; Haywood et al. 1997) would account for the lack of old sources in medium and deep surveys. In this context, Chandra and XMM-Newton surveys could be able to distinguish these two possible scenarios, thanks to their high sensitivity (Micela 2003).

The XMM-Newton Bright Serendipitous Survey (XBSS) was conceived with the aim of complementing the results obtained by medium and deep X-ray surveys. It contains two flux-limited samples of serendipitous XMM-Newton sources at galactic latitudes $|b|>20^{\circ}$ (see Fig. 1): the XMM Bright Source Sample (BSS) containing the sources detected in the energy range $0.5-4.5 \mathrm{keV}$ with a count-rate $\geq 10^{-2} \mathrm{cnt} \mathrm{s}^{-1}$ in EPIC-MOS2, and the XMM Hard Bright Source Sample (HBSS) with sources revealed in the energy range 4.5-7.5 keV with count-rate $\geq 2 \times 10^{-3} \mathrm{cnt} \mathrm{s}^{-1}$ (see Della Ceca et al. 2004, for details). For a Raymond-Smith spectrum with temperature $0.7 \mathrm{keV}$, typical of an active coronal source, the count-rate limit in the two chosen bands corresponds to an unabsorbed flux limit of $6.8 \times 10^{-14} \mathrm{erg} \mathrm{cm}^{-2} \mathrm{~s}^{-1}$ and $3.7 \times 10^{-13} \mathrm{erg} \mathrm{cm}^{-2} \mathrm{~s}^{-1}$, respectively. With this sensitivity, the XBSS complements deeper XMM-Newton and Chandra surveys with fluxes ranging from $10^{-14}$ to $10^{-15} \mathrm{erg} \mathrm{cm}^{-2} \mathrm{~s}^{-1}$.

The total area covered by the survey at the flux limit is 28.10 sq. deg. for the BSS (25.17 for the HBSS). The complete sample contains $400 \mathrm{X}$-ray sources, 389 sources belonging to the BSS. Up to now $\sim 90 \%$ of the BSS sources have been observed spectroscopically. Fifty-eight of these sources have been identified as stars, corresponding to $15 \%$ of the BSS sample. In the following we only refer to the BSS as our sample, since all the stars belong to it, while only two belong to the HBSS, which is largely dominated by extragalactic sources.

Thanks to the XMM's large effective area, the BSS is unique since it is the only survey of this kind where X-ray spectroscopy can be obtained for all the stars detected. The other particularity of the BSS is that it is unbiased at the flux limit; i.e., no a priori assumption has been made when selecting the sample. This makes the results representative of the entire solar neighbourhood. In addition, we should have detected all the young stars in the field of view. With the sensitivity of the BSS, X-ray sources with $L_{X} \sim 10^{29} \mathrm{erg} \mathrm{s}^{-1}$ (typical of young stellar coronal sources) could be observed at distances up to $110 \mathrm{pc}$ (see Sect. 4.1 for a more detailed description). Assuming a scale height of $100 \mathrm{pc}$ for the young $(0.01-0.1 \mathrm{Gyr})$ stellar population, our sample should contain the large majority of the young stars in the field of view. 

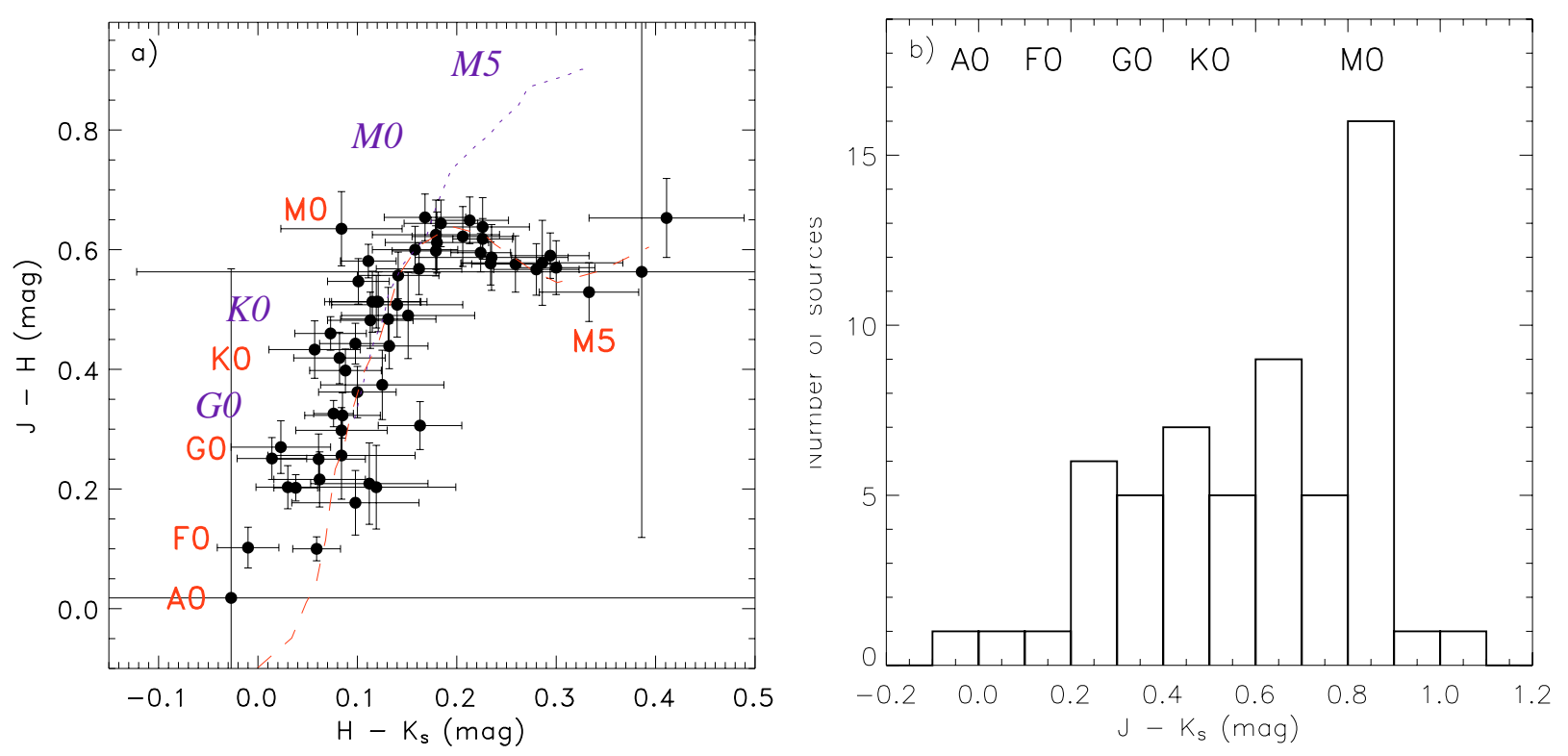

Fig. 2. a) Colour-colour NIR diagram for the stars in the BSS. Dashed and dotted lines are the main-sequence track and the giant branch defined by Bessell \& Brett (1989) transformed here to the 2MASS system (see text). Spectral types of the main sequence are labelled with roman font, at the corresponding $J-H$ value but offset in $H-K_{\mathrm{s}}$. Similarly, italic font has been utilised for labelling the spectral types of the giant branch. b) $J-K_{\mathrm{s}}$ distribution of the sources in a).

Here we carry out an analysis of the X-ray spectra of the 58 sources $^{1}$ identified as stars in the BSS (see Della Ceca et al. 2004, for details on the survey strategy and sample selection), as well as a study of their population, e.g. their distribution in spectral type and age, computed using the Galactic model XCOUNT. In Sect. 2 we determine the optical and infrared counterparts of the X-ray sources. In Sect. 3 we give the results of the analysis of the X-ray data. A discussion of the comparison between model predictions and observations is carried out in Sect. 4. Concluding comments are given in Sect. 5.

\section{Cross identification}

Infrared counterparts of our X-ray sources have been identified from the $2 \mathrm{MASS}^{2}$ database. For the cross identification, a search radius of 18 arcsec was adopted bearing in mind the 15.1 arcsec XMM-Newton mirror $p s f$ half energy width, the 1-2 arcsec uncertainty in the absolute position of a source in the EPIC detectors and the astrometric accuracy of $\sim 0.2$ arcsec of the 2MASS catalogue. Note that Della Ceca et al. (2004) estimate a positional error of the X-ray sources of the XBSS of 6 arcsec. The results of our cross identification of the stellar sample with the 2MASS database are in agreement with these errors. All the infrared counterparts are found within a radius of 8 arcsec from the centroid of the X-ray source, $95 \%$ with offset $\leq 4$ arcsec. A cross identification with the SIMBAD ${ }^{3}$ database and Tycho-2 catalogue was also carried out in order to identify obvious

1 Fifty six of them are given in (Della Ceca et al. 2004). The new stars identified since then are XBS J040744.6-710846 and XBS J122655.1+012002.

2 The Two Micron All Sky Survey is a joint project of the University of Massachusetts and the Infrared Processing and Analysis Center/California Institute of Technology, funded by the National Aeronautics and Space Administration and the National Science Foundation.

${ }^{3}$ SIMBAD Astronomical Database is operated at the CDS, Strasbourg, France. optical counterparts and to verify the spectral types of the infrared counterparts.

Of the 58 stars identified to date in the BSS, 29 have an optical counterpart in SIMBAD, while the whole sample has been cross identified with 2MASS. The infrared and optical counterparts are listed in Table 2, together with the $J H K_{\mathrm{s}}$ colour information from 2MASS. Right ascension and declination of the infrared sources and positional offset between the X-ray sources and their 2MASS counterparts are also given. In Fig. 2a we show the $J-H$ vs. $H-K$ colour-colour diagram for our sample and the main sequence track as defined by Bessell \& Brett (1989), adapted here to the 2MASS photometric system following Sect. 4 of the Explanatory Supplement to the 2MASS All Sky Survey ${ }^{4}$, along with the giant branch (Bessell \& Brett 1989). All the stars in the sample are on the main sequence track, which coincides with the giant branch for spectral types G0-M0. Nevertheless, two exotic objects were optically identified (see Table 2): the wide binary system WD 1631+78 (XBS J162911.1+780442) and the cataclysmic variable BL Hyi (XBS J014100.6-675328). The former is a binary system made up of a white dwarf and an active M4.5 dwarf (e.g. Cooke et al. 1992) which likely causes the observed X-ray emission (see Sion et al. 1995). The latter is a well-known polar - a binary system formed by a white dwarf and a pre-main sequence red dwarf - where the red spectral energy distribution of the companion dominates the overall colour (see Tovmassian et al. 2001; Hoard et al. 2002; Wachter et al. 2003; Caccianiga et al. 2004).

We used the infrared colours of the 2MASS counterparts for determining the spectral type of the sources, which will be used in comparing observations with model predictions (Sect. 4). For counterparts with $J-H \leq 0.6 \mathrm{mag}$. and $H-K_{\mathrm{S}} \leq 0.15 \mathrm{mag}$., we used $J-H$, while $H-K_{\mathrm{S}}$ colours were used for the rest of the sources. The reason for such a division is that the $J-H$ index is

\footnotetext{
${ }^{4}$ The 2MASS Explanatory Supplement is available on the web page: http://www.ipac.caltech.edu/2mass/releases/ allsky/doc/explsup.html
} 

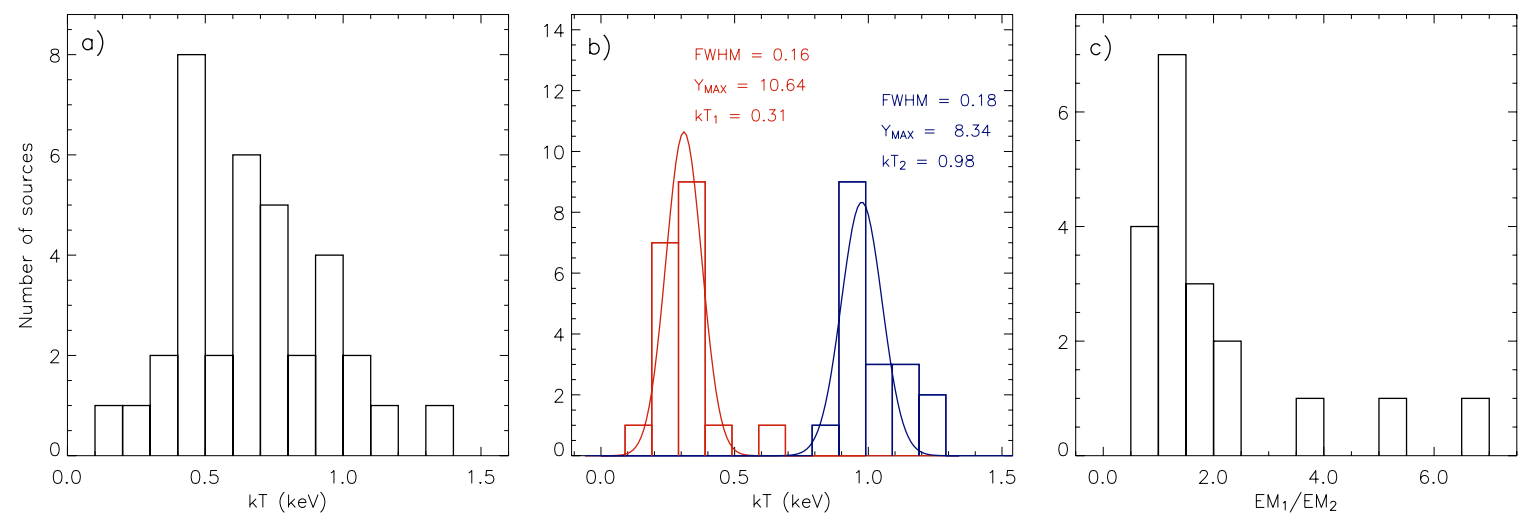

Fig. 3. a) Temperature distribution for the sources where a 1T-model has been fitted. b) Distribution of $T_{1}$ and $T_{2}$ of the stars where two thermal components have been fitted. c) Distribution of $E M_{1} / E M_{2}$ of the stars in Fig. b.

adequate for spectral classification of A to early-K stars but not for late-K and $\mathrm{M}$ dwarfs, where the differences in $J-H$ are very small. For them, the $H-K_{\mathrm{S}}$ index is more accurate (see Fig. 2a). Possible reddening has not been taken into account. However, no reddened sources are identified when comparing infrared colours with the optical ones from Tycho-2, for the sources in common. Figure $2 \mathrm{~b}$ shows the $J-K_{\mathrm{S}}$ distribution of the infrared counterparts. Sixty-four per cent of the sources (including both binary systems containing a white dwarf companion) are $\mathrm{K}-\mathrm{M}$ stars; the rest are yellow stars - spectral types F-G - with the exception of XBS J224833.3-511900, with colours typical of a B dwarf but with very large errors, XBS J080309.8+650807, and XBS J051617.1+794408 for which we obtain two possible counterparts with offsets of 2.3 and 7.1 arcsec, respectively. The photometry of the closest counterpart is very uncertain (flag = AEE in the 2MASS) and no conclusions about its spectral type can be inferred from it. Moreover, the 2MASS detection could be doubtful because of the proximity of the other source. Thus, for our study we have chosen the counterpart at 7.1 arcsec.

For 23 of our sources, we have optical spectra from the optical identification campaigns. In most cases, spectral types determined using this information agree well with those inferred from the infrared colours. Note that typical errors in the colours of these sources are $0.04 \mathrm{mag}$, typically equivalent to $2-3$ spectral subtypes. In Table 2 we give the results of the spectral classification using the information of both the infrared colours and the optical spectra. Based on infrared colours, only two sources seem to be significantly reddened: XBS J095955.2+251549 and XBS J122942.3+015525. The difference between the infrared and the optical inferred spectral types in both sources is higher than acceptable for their infrared colour errors. Another source (XBS J074359.7+744057) shows discrepancy in the spectral classification. In this case, the infrared colour is more typical of a late-G or early-K star while the optical spectrum is clearly from a late-K dwarf. Likewise, the two stellar systems containing a white dwarf are clearly identified, since the low-mass companion dominates in the infrared range while the white dwarf is dominant in the optical spectrum.

\section{X-ray properties of the sample}

We carried out a spectral analysis of all the 58 X-ray stellar sources using data from the EPIC camera and the XSPEC spectral fitting package (Arnaud 1996, 2004). When possible, data from the PN, MOS1, and MOS2 detectors were used. In some cases, no image from EPIC-PN was collected due to both the source position in the field of view and the camera configuration - note that the source sample was defined using only the data from the EPIC-MOS2 detector (see Della Ceca et al. 2004, for details) without restrictions on the EPIC-PN configuration - and only MOS data have been used.

\subsection{X-ray spectra and temperatures}

For the analysis, we adopted the APEC database, which contains the relevant atomic data for both continuum and line emission (Smith et al. 2001) included in XSPEC software. Interstellar absorption has been taken into account using the interstellar photoelectric absorption cross-sections of Morrison \& McCammon (1983), also available in XSPEC. A two-temperature model was used for those stars where a 1T-model did not account for the hard tail of the spectrum, i.e. the least-square solution to the 1T-model gives unsatisfactory $\chi^{2}$ goodness-of-fit values. Free parameters of the model are the $N_{\mathrm{H}}$, the global abundance scaled on solar values from Anders \& Grevesse (1989), and the temperature and the emission measure $(E M)$ for each thermal component. For the stars HD 32558 and CD-39 7717B, a 3T-model was used to obtain an accurate fitting to the hard tail of the X-ray spectrum.

The results of the fits are shown in Table 3: for each star we give the value of the coronal temperature(s), the emission measures rate $\left(E M_{1} / E M_{2}\right)$ when two thermal components are fitted, the global abundance $\left(Z / Z_{\odot}\right)$, the interstellar absorption $\left(N_{\mathrm{H}}\right)$, the reduced $\chi^{2}$ value and associated degrees of freedom of the fit, the total net counts in the EPIC (MOS + PN) chips, and the unabsorbed flux in the energy range $0.5-10 \mathrm{keV}$. The error ranges refer to the $90 \%$ confidence region, or $2.706 \sigma$. For those sources with known parallax, the X-ray luminosity $\left(\log L_{\mathrm{X}}\right)$ was determined (see Table 4). The results show a sample dominated by active stars - even though it is highly biased - with $\log L_{X}$ values typical of those observed in the Hyades and the Pleiades. Furthermore, two of the sources (J001002.4+110831 and J123600.7-395217, see Table 2) were identified with post-T Tauri stars.

In Fig. 3 we plot the results in terms of temperature and $E M$ distributions. For the sources where a $1 \mathrm{~T}$-model is adequate for reproducing the spectra, a broad spread of temperature is present (see Fig. 3a). For them, no definite conclusions can be reached. Some of the sources showing a low coronal temperature have a very low $S / N$ and little can be said about their temperature stratification except that the X-ray spectra is dominated by this temperature. Several stars with a mean coronal 
temperature $k T$ in the range $[0.5,0.8] \mathrm{keV}$ show high $S / N$, suggesting that only one thermal component is present in the corona. However, this is common only in F-type stars. In addition, a considerable fraction of the sources $(25 \%)$ have a mean coronal temperature over $0.8 \mathrm{keV}$. This suggests the presence of two unresolved thermal components in their X-ray spectra, the hotter component contributing more than the cooler one. On the other hand, the spread in the temperature distributions of both thermal components of the sources where a 2T-model has been used is noticeably lower (see Fig. 3b). A Gaussian curve has been fitted to both peaks in the temperature distribution obtaining a $F W H M$ of 0.15 and $0.19 \mathrm{keV}$, which is compatible with the estimated errors (see Table 3 ). These results indicate that the corona of these sources can be described by two thermal components, a first one peaking at $0.31 \mathrm{keV}$ and a second one at $0.98 \mathrm{keV}$. Similar results have been found by a number of authors (Vaiana 1983; Schmitt 1984; Majer et al. 1986; Schmitt et al. 1990) for late-type stars observed with the Einstein Imaging Proportional Counter (IPC; Gorenstein et al. 1981). In particular, Schmitt et al. (1990) found that a 2T-model with $k T_{1} \sim 0.22 \mathrm{keV}$ and $k T_{2} \sim 1.37 \mathrm{keV}$ is more adequate than the classical 1Tmodel for describing the observed X-ray spectrum of most of the dwarfs in an extensive sample of late-type stars. Although Schmitt (1984) and Majer et al. (1986) find that two such temperature components correspond to regions of the IPC's peak sensitivity, Schmitt et al. (1990) conclude that the dichotomy is not due to an artifact of their analysis, but must represent an intrinsic property of the coronal spectra of moderately active late $\mathrm{K}$ and M dwarfs. Confirming these results, Briggs \& Pye (2003) used XMM-Newton observations to show that the temperature structure in the quasi-steady coronae of solar-like Pleiads can be reproduced by two thermal components with approximately equal emission measures.

The values of the two temperatures we found for the stars in which a 2T-model is needed are compatible with the ones obtained by Briggs \& Pye (2003) in non-saturated K stars in the Pleiades (see their Fig. 5). The contribution of both components of our sources to the X-ray spectrum also agrees with their results for $\mathrm{K}$ stars. In Fig. 3c we plot the distribution of the ratio of the emission measures $\left(E M_{1} / E M_{2}\right)$ of the sources. The data show a peak around 1.0-1.5, with a spread that accounts for the different contributions of the two thermal components in each coronal source. Only four stars present higher contribution from the hotter thermal component, all them containing more than 3000 counts. On the other hand, three sources show $E M_{1} / E M_{2}$ values higher than 3 , i.e. their spectrum is clearly dominated by the cooler component. All these stars contain less than 1000 counts. Although one is tempted to look for a correlation between the number of counts and $E M_{1} / E M_{2}$ - since the higher the coronal activity, the hotter the corona of the source and the higher its count rate - no clear conclusions can be inferred from these results, since some of the sources with high $S / N$ do not show higher contribution from the hotter component.

The values of $N_{\mathrm{H}}$ determined from the fitting of the X-ray spectra are typical of nearby stars for the majority of our sources. However, nine of them (15\% of our sample) show a $N_{\mathrm{H}}$ that is significantly higher than the Galactic column density. The high $N_{\mathrm{H}}$ derived implies that these stars might be surrounded by diffuse interstellar material left over from its formation, since all our stars are nearby (see Sect. 1). The total number of counts for some of these sources is very low so no conclusions can be inferred from the fitting. In these cases, the high value of $N_{\mathrm{H}}$ could be an artifact of the fitting procedure, and other solutions with similar goodness-of-fit values, but with different fitting parameters, could be obtained. Instead, at least two of the sources (XBS J074359.7+744057 and XBS J122942.3+015525) with high $N_{\mathrm{H}}\left(0.76 \times 10^{21}\right.$ and $2.73 \times 10^{21} \mathrm{~cm}^{-2}$, respectively) have also a high number of counts ( $\sim 700$ counts) and show evidence of local reddening in their infrared colours, when comparing them with the spectral type deduced from their optical spectrum (see Table 2). The source XBS J095955.2+251549, with $N_{\mathrm{H}}=0.76 \times 10^{21} \mathrm{~cm}^{-2}$, also shows a discrepancy between the spectral type deduced from the infrared colours and that determined from the optical spectrum. An $N_{\mathrm{H}}$ of $0.6-2.7 \times 10^{21} \mathrm{~cm}^{-2}$ (corresponding to $A_{V} \approx 0.3-1.3 \mathrm{mag}$., using the $\left(N_{\mathrm{H}} / A_{V}\right)_{\text {gal }}$ relation in Ryter 1996 and Vuong et al. 2003) is compatible with what is found in some young stars in the nearby stellar associations TW Hya, Tucana-Horologium, and $\beta$ Pic (e.g. Kastner et al. 2003; Zuckerman \& Song 2004), where the residual circumstellar material is expected to dominate the X-ray absorption. Thus, at least for these three sources, the high $N_{\mathrm{H}}$ seems not to be an artifact of the fitting process but the result of X-ray absorption by circumstellar material, indicative of a young star still close to its formation site. In particular, they could be weak-lined T Tauri stars (WTTS) that have already lost their disk, since they do not present any IR excess in the colour-colour diagram (Fig. 2a).

\subsection{X-ray variability}

The light curve of the sources in our sample was investigated with the aim of finding variations in their X-ray emission. A best extraction region was determined for each source taking their $p s f$ into account in order to contain the maximum number of photons coming from the source and the minimum from the background. Besides, a background light curve was extracted for background subtraction from a region of the field of view close to the source and free of other contaminating sources. Error bars were determined assuming Poissonian distribution of the photons collected by the chip in both source and background regions.

For our study we utilised a Kolmogorov-Smirnov test. We have chosen a (significance) probability $P>0.05$ for discarding the source as variable, which means we can reject the hypothesis that the source is constant - i.e. the X-ray photons came from the source in a constant rate - at the $95 \%$ confidence level. Of the 58 sources composing the BSS, 39 fulfill this condition and other 3 are in the range $90-95 \%$ of confidence level. This means that $67 \%$ of the sources of the sample are variables. Fifteen of these sources show at least one flare during the observations, corresponding to $26 \%$ of the sample.

The observed curves show very different patterns related with distinct processes (see Fig. 4). For instance, XBS J051617.1+794408 and XBS J110320.1+355803 present solar-like flare events. These sources have been identified (see Sect. 2) with the stars HD 32558 and HD 95735, respectively. The latter is a well-known flare M2 dwarf. In contrast, little is known about HD 32558. SIMBAD database classifies it as F8, and photometric data from Tycho-2 confirm it is an F star. In a study of the X-ray variability of Pleiades late-type stars, Marino et al. (2003) show that F-G Pleiades stars are variable on medium or long time scales (days-months). Short time (flare) variability (i.e. time scales of hours) is also observed, although it is more common in $\mathrm{dK} 3-\mathrm{dM}$ stars. Comparing the time X-ray distribution functions with those of field stars, the authors demonstrate that short time variability is more common in Pleiades F-G stars than in field dwarfs of similar spectral types. Thus, the observation of this X-ray flare suggests it is a young star, although we cannot discard the existence of a cooler companion producing the event. Other stars show light 

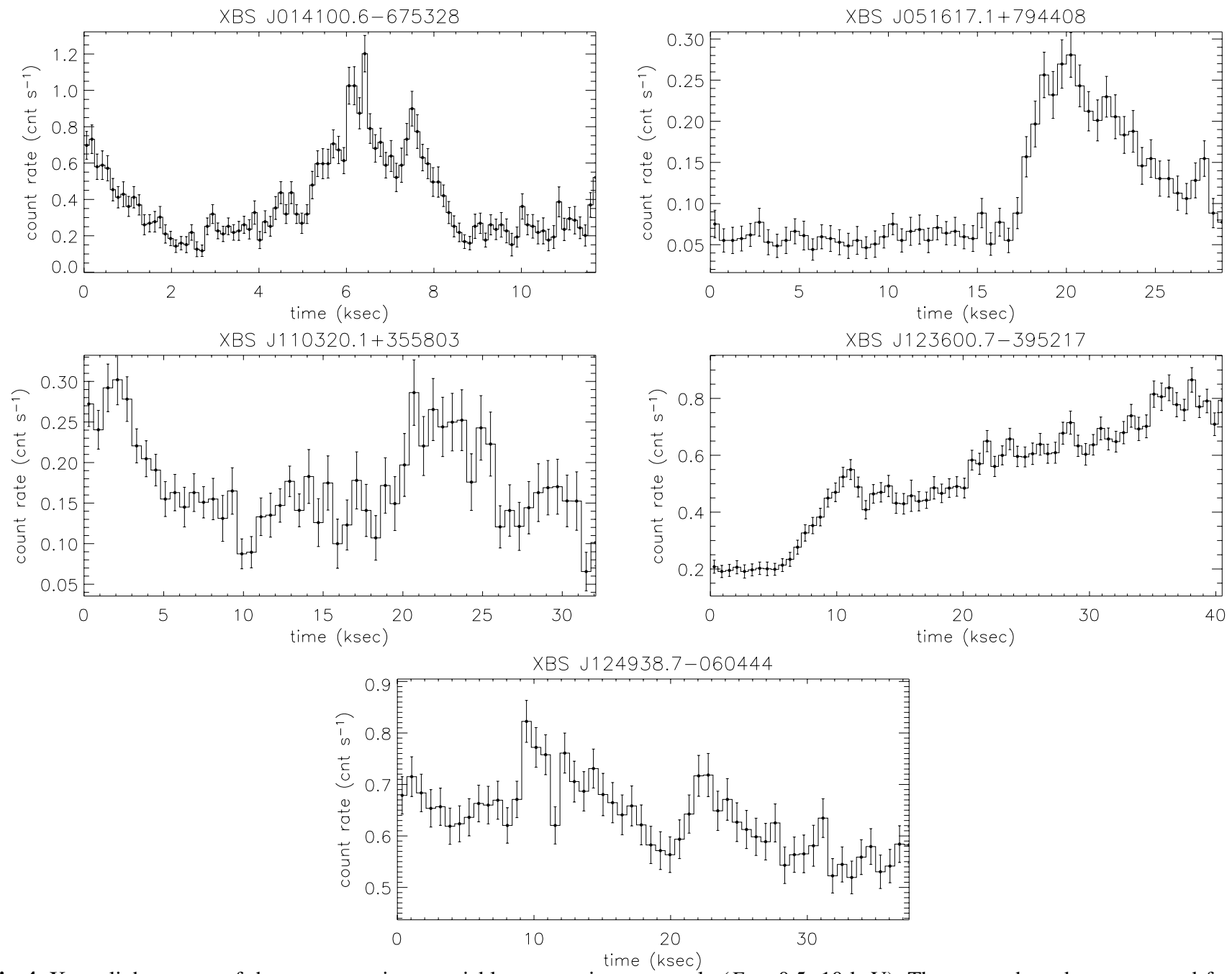

Fig. 4. X-ray light curves of the most prominent variable sources in our sample $(E=0.5-10 \mathrm{keV})$. The curves have been corrected for background (see text for details), GTI, and dead-time effects. EPIC-PN data were used except for XBS J014100.6-675328 (BL Hyi), where we chose EPIC-MOS1 data to also show the first emission decay.

curves that may be produced by quite different physical processes. Thus, the continuous enhancement observed in the light curve of XBS J123600.7-395217 could be related to accretion processes in T-Tauri class objects. The source was identified (see Sect. 2) with the very young system CD-39 7717 (TWA-11), made up of a T-Tauri star with spectral type A0 and an M2.5 young star and member of the TW Hya association (TWA). The A0 star contains a debris system that is believed to have a terrestrial planetary system (Low \& Smith 2005). The M companion is a PMS. The resolution of the X-ray observation does not permit the components to be separated, and not much can be said about which star the X-ray emission comes from or whether it is the sum of the emission of both. Nevertheless, the A0 component is known to be under-luminous in X-rays compared to the average observed X-ray emission for the rest of the TWA members (e.g. Low \& Smith 2005), and so the observed emission is likely to come from the secondary. We used a Chandra ACIS-S observation from the archive in which the system was serendipitously observed (Obs. ID: 2150) in order to resolve both components. The position of the X-ray source in ACIS-S confirms that the emission comes from the $\mathrm{M}$ star. This agrees with the results of the cross identification (see Sect. 2) where the source is identified with the $\mathrm{M}$ star.
The other two examples of peculiar X-ray variability are (i) XBS J124938.7-060444, identified with the chromospheric active binary IM Vir, which X-ray emission shows a clear modulation with time, and (ii) XBS J014100.6-675328 (BL Hyi) which show an energetic flaring event decaying in one hour. This is a well-known polar (e.g. Tovmassian et al. 2001) observed in an accretion state.

\section{Model predictions}

\subsection{Model description and assumptions}

The X-ray Galactic model XCOUNT (Favata et al. 1992; Micela et al. 1993) was used for predicting the number and properties of the coronal sources expected in the BSS. The model is based on the stellar spatial densities of the Bahcall \& Soneira model (Bahcall \& Soneira 1980; Bahcall 1986). Although the Bahcall \& Soneira model includes both an exponential disk and a spheroid, XCOUNT considers only the disk component due to the low X-ray luminosity of spheroid stars (see Favata et al. 1992). With the sensitivity of the BSS, active coronal sources with $L_{\mathrm{X}} \sim 10^{29} \mathrm{erg} \mathrm{s}^{-1}$ could be observed at distances up to $\sim 110 \mathrm{pc}$, while the maximum distance at which a typical quiet star with $L_{\mathrm{X}} \sim 10^{27} \mathrm{erg} \mathrm{s}^{-1}$ might be visible is $\sim 10 \mathrm{pc}$. Then, the 
approximation to a disk is quite realistic since we are observing only stars in our neighbourhood. Note that the scale height adopted by Bahcall (1986) is $350 \pm 50$ pc for main sequence stars and $250 \pm 100$ for giants. On the other hand, our model is not simply a disk. The spatial distributions of the disk population in the original optical model were modified to separate several distinct subpopulations of A-M stars of different ages. This introduces an explicit dependence of the stellar spatial distribution and density on stellar age (Micela et al. 1993).

Different scale heights were adopted for distinct age ranges: 100,200 , and $400 \mathrm{pc}$ for $0.01-0.1,0.1-1$, and $>1$ Gyr respectively. They were determined taking the results of Basu \& Rana (1992) into account. Assuming the Galaxy to be formed by a disk that is isothermal in the z-direction (Wielen \& Fuchs 1983), the scale height $(H)$ is correlated with the velocity dispersion $\left(\sigma_{\mathrm{z}}\right)$ as $H \propto \sigma_{\mathrm{z}}^{2}$. At the same time, the velocity dispersion is proportional to $t^{\mathrm{n}}$ (Villumsen 1985), so the scale height is proportional to the age as $H \propto t^{2 \mathrm{n}}$. The value of $n$ must be determined from empirical data. In particular, Basu \& Rana (1992) obtained $2 n=\frac{2}{3}$ using data given in Robin \& Crézé (1986).

Three different X-ray luminosity functions (XLFs) were used for the distinct age ranges: the youngest stars with ages 0.01-0.1 Gyr are assigned the ROSAT XLF of the Pleiades members (Micela et al. 1996); intermediate-age stars with ages 0.1-1 Gyr are assigned the ROSAT XLF of the Hyades (Stern et al. 1995); and the older ones ( $t>1 \mathrm{Gyr})$ are assigned the XLF of Einstein studies of nearby old-disk stars (Schmitt et al. 1985; Maggio et al. 1987; Barbera et al. 1993). The XLFs were appropriately converted to the bandpass of the observations, assuming a given input spectrum (see Favata et al. 1992, for details). Such a set of XLFs permits evaluation of the contribution to the total number of stars of different age populations without losing information about the assumed optical Galaxy model (Favata et al. 1992).

In general, predicted optical star counts are not strongly influenced by age distribution of the disk populations (Micela et al. 1993) because both optical luminosity and colour do not change substantially with stellar evolution during the main sequence lifetime. On the contrary, due to the high dependence of the X-ray luminosity with age (Micela et al. 1985, 1988; Guedel et al. 1997; Feigelson \& Montmerle 1999), the assumed age distribution significantly influences the predicted X-ray source counts. Here we have performed model computations with the three age subgroups having relative density on the plane derived from an exponential law:

$\Psi=A \mathrm{e}^{-t / \tau}$

where $\tau$ can take the values $\infty, 15,5$, and -15 Gyr, corresponding to constant, slowly decreasing, rapidly decreasing, and slowly increasing stellar birthrate, respectively, according to the results by Basu \& Rana (1992).

\subsection{XCOUNT model results}

In Fig. 5 we show the predictions of the model with relative density distributions for the three age subgroups given by the above mentioned stellar birthrates. The numbers are summarised in Table 1 . The 2MASS $J-K_{\mathrm{S}}$ colours of the observed $\mathrm{X}$-ray sources infrared counterparts have been converted to the Bessell \& Brett photometric system - utilised by the model using the transformation given in the Explanatory Supplement to the 2MASS All Sky Survey, for comparing observations with predictions.

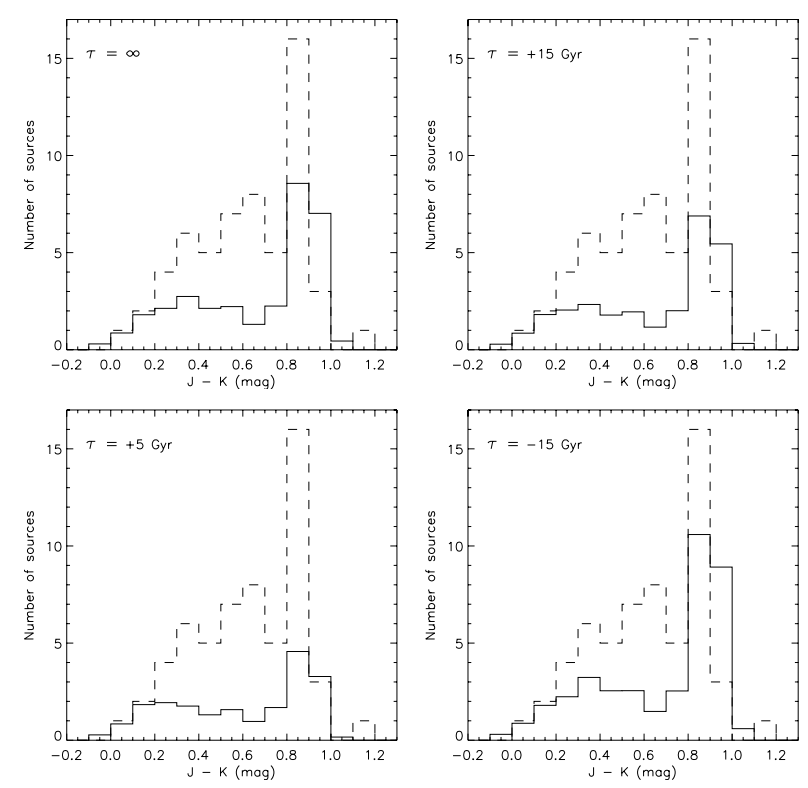

Fig. 5. Colour distributions predicted by XCOUNT (continuous line) compared with the observed one (dashed line) for different stellar birthrates assuming an exponential law $\Psi=A \mathrm{e}^{-t / \tau}$.

A first inspection of the results suggests the stellar birthrate is an increasing function of time since the total number of predicted sources is in better agreement with the observed ones when using $\tau=-15 \mathrm{Gyr}^{5}$. However, the number of predicted $\mathrm{M}$ stars using this birthrate is quite above the observed one, even taking the error propagation in the model into account. In contrast, predictions made for $\mathrm{M}$ stars using both constant or slowly decreasing birthrates are quite in agreement with the observations. Note as well that the slowly increasing stellar formation rate (SFR) gives very similar results to those of the constant birthrate.

On the other hand, the model does not account for the number of observed FGK stars using any of the birthrates analysed here. An excess of yellow stars is also observed in other shallow surveys (Favata et al. 1988; Micela 2003), which cannot be reproduced by Galactic models using smooth shapes of the SFR. In particular, Favata et al. (1993) and Sciortino et al. (1995) confirm the excess of yellow stars in the Extended Medium Sensitivity Survey of Einstein (EMSS, Gioia et al. 1990) as being due to young stars. Similarly, Micela et al. (2006) find an excess of FGK stars in the ROSAT North Ecliptic Pole survey (NEP, Henry et al. 2001). This result excludes scenarios with decreasing stellar birthrate, but does not discard continuous SFR with one or more bursts. Studies of the IMF (Miller \& Scalo 1979) and metallicity distribution in F stars (Twarog 1980; Carlberg et al. 1985) show that the SFR has basically remained constant over the age of the Galactic disk, or that it might have been smaller in the past (Miller \& Scalo 1979). Our results show that the constant and the slowly increasing birthrate hypothesis are nearly equivalent, and none of them reproduce the counts for yellow stars.

\footnotetext{
${ }^{5}$ Note that typical values for the relative error on the predicted source counts - for the error propagation of the statistical errors associated with the X-ray luminosity functions - are in the range 15-30\% (Favata et al. 1992). Taking this and the $\sqrt{N}$ statistical uncertainties in the model simulation into account (Feigelson et al. 2004) we estimate a range of $19-43,15-35,12-28$, and $24-51$ predicted sources for stellar birthrates with $\tau=\infty,+15,+5$, and -15 Gyr respectively.
} 
Table 1. XCOUNT predicted X-ray counts using different stellar birthrates, compared with the observations.

\begin{tabular}{lcccccccc}
\hline \hline & B & A & F0-F6 & F7-G & K & M0-M5 & Total & Range $^{\ddagger}$ \\
\hline Observed $^{\ddagger}$ & 1 & 2 & 9 & 9 & 21 & 14 & 56 & \\
Constant birthrate $(\tau=\infty)$ & - & 2 & 3 & 5 & 7 & 14 & 31 & $19-43$ \\
Slowly decreasing birthrate $(\tau=15 \mathrm{Gyr})$ & - & 2 & 3 & 4 & 6 & 11 & 26 & $15-35$ \\
Rapidly decreasing birthrate $(\tau=5 \mathrm{Gyr})$ & - & 2 & 3 & 3 & 5 & 7 & 20 & $12-28$ \\
Slowly increasing birthrate $(\tau=-15 \mathrm{Gyr})$ & - & 2 & 3 & 6 & 8 & 18 & 37 & $24-51$ \\
\hline
\end{tabular}

$\dagger$ Range of total number of stars predicted by the model, for each stellar birthrate. This value has been estimated taking the relative error on the predicted source counts and the statistical uncertainties in the model simulation into account.

\# The two binary systems containing a white dwarf (see Sect. 2) have been discarded for the comparison.
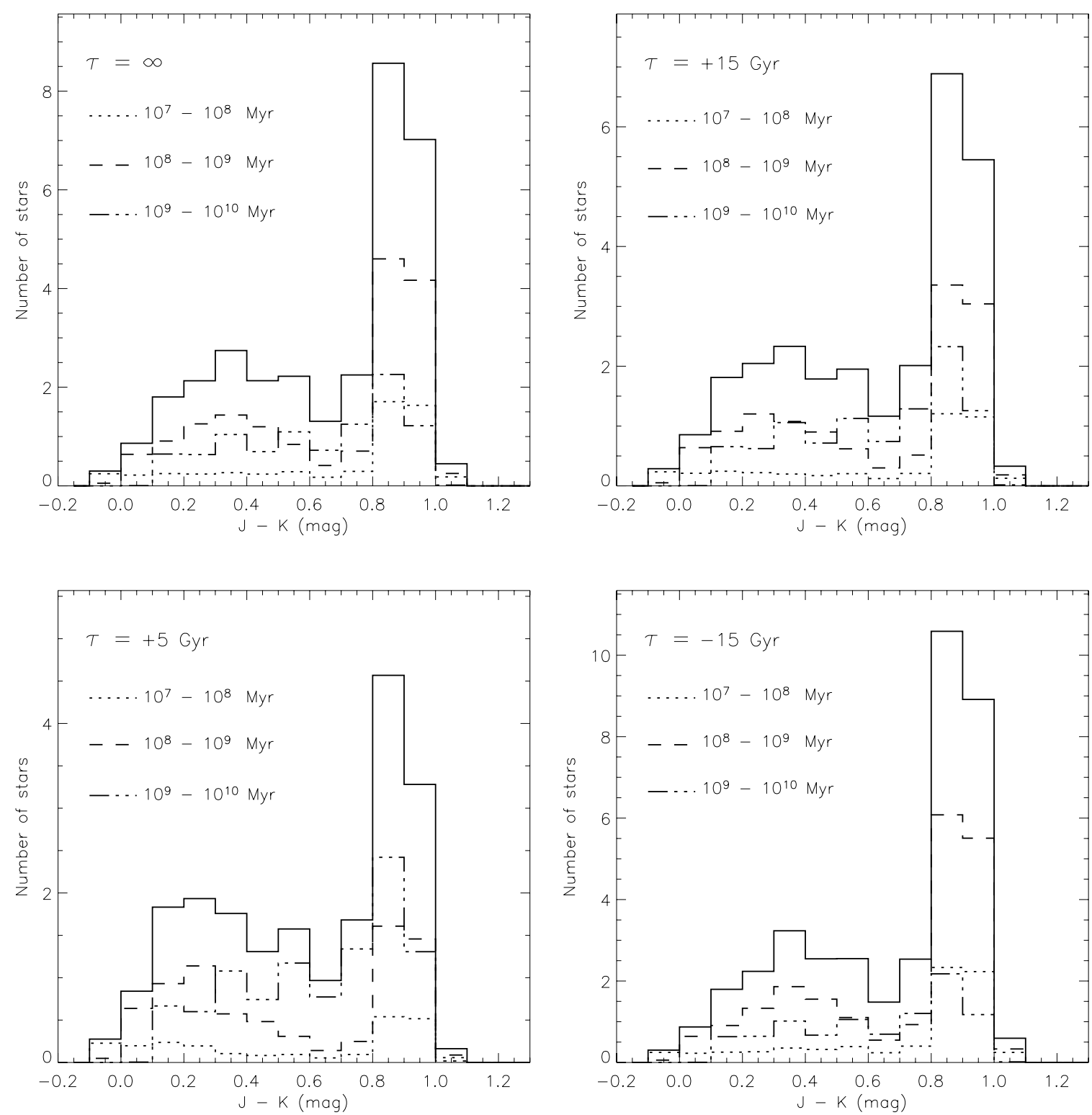

Fig. 6. Colour distributions predicted by XCOUNT for different stellar birthrates assuming an exponential law $\Psi=A \mathrm{e}^{-t / \tau}$. The contribution of different age-subgroups to the total source counts is shown in each plot.

\subsection{The nature of the FGK excess}

From the results presented above one can argue that no smooth stellar birthrate can account for the observed distribution of stars. Although the total number of sources can be reproduced quite well using a slowly increasing SFR, the observed and predicted distributions in spectral type are different: there is an excess of stars with spectral types $\mathrm{F}-\mathrm{K}$ in the observations and a lack of observed $\mathrm{M}$ stars that compensates the excess of FGK stars. Taking into account that using different scale heights makes the total number of stars vary but not the relative distribution in spectral types, and assuming that the stellar birthrate is indeed smooth - no definite evidence of star burst are observed in 
Table 4. Unabsorbed X-ray luminosity and emission measure for the stars in the sample with known parallax.

\begin{tabular}{lrcc}
\hline \hline $\begin{array}{l}\text { Star } \\
(\mathrm{XBSS} \ldots)\end{array}$ & $\begin{array}{c}\text { Distance } \\
(\mathrm{pc})\end{array}$ & $\begin{array}{c}\log E M^{*} \\
\left(\mathrm{~cm}^{-3}\right)\end{array}$ & \multicolumn{1}{l}{$\begin{array}{c}\log L_{\mathrm{X}} \\
\left(\mathrm{erg} \mathrm{s}^{-1}\right)\end{array}$} \\
\hline J001002.4+110831 & 99.60 & 54.30 & 29.94 \\
$\mathrm{~J} 001749.7+161952$ & 46.17 & 52.33 & 28.56 \\
$\mathrm{~J} 080309.8+650807$ & 114.02 & 52.77 & 29.17 \\
$\mathrm{~J} 110320.1+355803$ & 2.55 & 51.21 & 26.49 \\
$\mathrm{~J} 123600.7-395217$ & 67.07 & 53.56 & 29.88 \\
$\mathrm{~J} 212635.8-445046$ & 114.16 & 53.81 & 29.37 \\
J213840.5-424241 & 77.28 & 52.49 & 28.71 \\
$\mathrm{~J} 224833.3-511900$ & 39.75 & 52.73 & 28.65 \\
$\mathrm{~J} 231541.2-424125$ & 99.11 & 52.67 & 28.91 \\
\hline
\end{tabular}

* For those stars where more than one thermal component has been fitted to the X-ray spectrum, $E M$ is the emission measure of the first component.

the recent history of the disk (see Twarog 1980; Carlberg et al. 1985, but see also Barry 1988) - the discrepancy between predictions and observations must be due to a stellar population not accounted for by the model.

In Fig. 6 we show the relative contribution of the stars in the three age ranges to the total stellar counts, when using SFR with $\tau=\infty,+15,+5$, and -15 Gyr. In the cases of rapid or slowly decreasing birthrates, the X-ray population is dominated by intermediate-age and old stars with almost the same contribution. However, when using a constant or a slowly increasing SFR, the intermediate age sources are clearly dominant. In all the cases, the young stars give a small contribution (less than 20\%). The XCOUNT model allows the determination of the number of sources of any type expected in an X-ray observation given their spatial distribution and luminosity function, since they are treated as external parameters (Favata et al. 1992). Thus, we used XCOUNT to predict the number of disk giant stars observed in the BSS, using the spatial distribution of the Bahcall and Soneira model, a scale height of $250 \mathrm{pc}$ and a coronal temperature of $1.1 \mathrm{keV}$ (Schmitt et al. 1990). The result is that no more than 1 giant is expected to be detected in the sample, which discards giants as the cause of the observed excess of yellow stars.

Bearing these results in mind, we infer that only two populations can be responsible for the excess in the BSS: active binaries - RS CVn and BY Dra systems - and/or very young objects. In Fig. 7 we plot the $\log N-\log S$ predicted at a high latitude for the four stellar birthrates, compared with observations in the fields observed with the medium filter (the results with the other filters are equivalent). The observed cumulative curve is clearly above the predictions. At low count rates, the curve is almost parallel to the predicted $\log N-\log S$, but there is a bump at high count rates $\left(\geq 6 \times 10^{-2} \mathrm{~s}^{-1}\right)$ that could result from the presence of a population of sources with high X-ray luminosity and low scale height. This would show a scenario of recent stellar birthrate enhancement, which agrees with the results of the EMSS (Favata et al. 1993; Sciortino et al. 1995). On the other hand, if we assume the scenario with an increasing stellar birthrate to be correct as suggested by the better fit to the total number of stars (see Table 1) - we would be observing less $M$ stars than those predicted. A possible hypothesis for the observed excess of yellow stars in such a scenario with an increasing stellar birthrate and a lack of red stars is the existence of a number of X-ray M dwarfs in binary systems with a yellow primary.

\section{Discussion and conclusions}

We have studied the stellar content of the XMM Bright Serendipitous Survey in detail. The 2MASS counterparts have been identified for each one of the $58 \mathrm{X}$-ray sources. This unbiased stellar sample was characterised for coronal temperature, global abundance and column density, and a study of the light curves was carried out to reveal the X-ray variable population. Considering that the sample is unbiased, the results must be typical of the entire solar neighbourhood at the flux limit of the survey. This is the first time in which a complete X-ray spectral characterisation has been carried out for a survey of this kind, thanks to the XMM-Newton effective area. In general, the coronal temperature stratification of our sources is typical of moderately active stars. Their spectrum can be fitted using a $2 \mathrm{~T}$-model with $k T_{1}=0.32 \pm 0.08 \mathrm{keV}$ and $k T_{2}=0.98 \pm 0.09 \mathrm{keV}$. Only for two sources a third thermal component was necessary for fitting the hard tail of the spectrum. For other sources with low $\mathrm{S} / \mathrm{N}$ a $1 \mathrm{~T}$-model is adequate for reproducing the spectrum. The value of the temperature in these cases is not representative of the temperature stratification but only of the dominant thermal component. Of the 58 sources, 42 present variability in their light curves, corresponding to $67 \%$ of the whole sample. Fifteen of these sources ( $26 \%$ of the whole sample) showed at least one flare during the observations.

We performed a detailed comparison between the observed stars and the predictions computed using the XCOUNT model with four different stellar birthrates. From the comparison of the expected number of stars of each spectral type with that inferred from the 2MASS counterparts, we show that a decreasing stellar birthrate scenario is not probable since the total number of predicted stars is clearly below the observed one, even taking model uncertainties into account. A constant SFR can reproduce the number of $\mathrm{A}$ and $\mathrm{M}$ sources observed well, but still underestimates the total number of observed stars. In fact, an increasing birthrate seems more suitable to the BSS: the total number of predicted sources fit the observations bearing model uncertainties in mind, although it overestimates the total number of M stars. On the other hand, the excess of FGK stars cannot be reproduced using only a smooth stellar birthrate. Assuming that the stellar birthrate has indeed been smooth in the past billion years, we infer that the discrepancy between observations and predictions must be produced by a stellar population not directly taken into account in the model.

In the $\log N-\log S$ diagram, the cumulative curve of the BSS sources is above the predicted one for the four stellar birthrates analysed here. The BSS curve runs parallel to the predicted ones for low count rates, but a bump is clearly observed at higher count rates $\left(\geq 6 \times 10^{-2} \mathrm{~s}^{-1}\right)$. This again suggests the existence of a population with high X-ray luminosity and low scale height contributing to the sample. The high percentage of flare stars observed in the sample agrees with this hypothesis. Nevertheless, the lack of observed $\mathrm{M}$ stars with respect to the predictions when using the increasing stellar birthrate suggests another explanation: the existence of a number of X-ray $M$ stars in binary systems with a yellow primary.

We have shown that a scenario with a decreasing stellar birthrate is unsuitable for the observed number of stars and their distribution in spectral type. Furthermore, the situation with a constant SFR underestimates the total number of observed sources and does not seem to be appropriate. On the contrary, we show that a scenario with an increasing stellar birthrate can account for the total number of stars, and that the discrepancy in the distribution of the stars in spectral type between predictions 


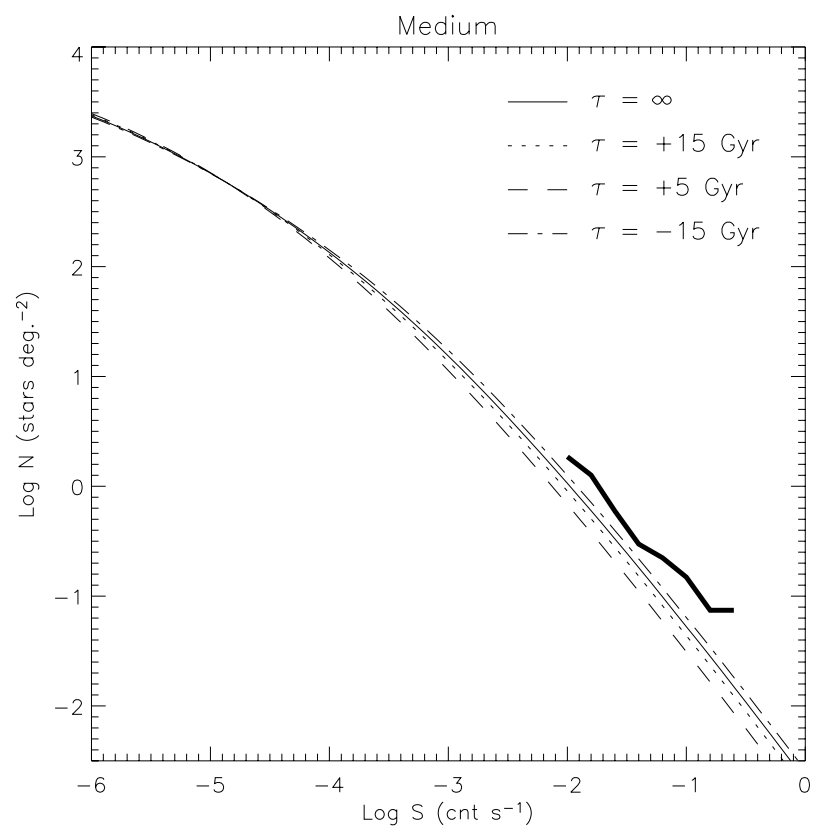

Fig. 7. $\log N-\log S$ predicted assuming $\tau=\infty,+15,+5$, and $-15 \mathrm{Gyr}$, compared with the BSS results (thick solid line) for the fields observed with the medium filter (energy band $0.5-4.5 \mathrm{keV}$ ). For the comparison we used a standard high latitude direction $b=60^{\circ}$.

and observations could be due to a population of young stars with low scale heights or to a number of $\mathrm{M}$ dwarfs in binary systems with a yellow primary.

Finally, we have shown that in several stars of the sample, some residual material from its formation could be left over. The value of $N_{\mathrm{H}}$ determined for these stars is compatible with the optical absorption and column density observed in stars of the young stellar associations TW Hya, Tucana-Horologium, and $\beta$ Pic. This agrees with the scenario with an increasing stellar birthrate and a population of young stars in the solar neighbourhood. These stars would be good targets for examining the evolution of gaseous circumstellar disks around young stars.

Acknowledgements. J. López-Santiago acknowledges support by the Marie Curie Fellowship contract No. MTKD-CT-2004-002769. The XMM BSS project received financial support by the Italian Space Agency (ASI), by the Ministero dell'Instruzione Nazionale, Università e Ricerca Scientifica (MIUR), and by the Italian Istituto Nazionale di Astrofisica (INAF). We also thank the TNG, ESO and Calar Alto Time Allocation Committee for a generous and continuous allocation of observing time during the last years. We thank Tommaso Maccacaro for his strong support of the XMM/Newton BSS project. This publication makes use of data products from the Two Micron All Sky Survey, which is a joint project of the University of Massachusetts and the Infrared Processing and Analysis Center/California Institute of Technology, funded by the National Aeronautics and Space Administration and the National Science Foundation. This research made use of the SIMBAD database, operated at the CDS, Strasbourg, France.

\section{References}

Anders, E., \& Grevesse, N. 1989, Geochim. Cosmochim. Acta 53, 197

Arnaud, K. 1996, in XSPEC: The First Ten Years, ed. G. H. Jacoby, \& J. Barnes, ASP Conf. Ser., 101, 17

Arnaud, K. 2004, XSPEC: Progress and Plans. In the American Astronomical Society, HEAD meeting 8, 16.29
Bahcall, J. N., \& Soneira, R. M. 1980, ApJS, 44, 73

Bahcall, J. N. 1986, ARA\&A, 24, 577

Baldi, A., Molendi, S., Comastri, A., et al. 2002, ApJ, 564, 190

Barbera, M., Micela, G., Sciortino, S., Harnden, F. R., \& Rosner, R. 1993, ApJ, 414,846

Barry, D. C. 1988, ApJ, 334, 436

Basu, S., \& Rana, N. C. 1992, ApJ, 393, 373

Bessell, M. S., \& Brett, M. 1988, PASP, 100, 113

Brandt, W. N., Alexander, D. M., Hornschemeier, A. E., et al. 2001, AJ, 122, 2810

Briggs, K. R., \& Pye, J. P. 2003, MNRAS, 345, 714

Caccianiga, A., Severgnini, P., Braito, V., et al. 2004, A\&A, 416, 901

Carlberg, R. G., Dawson, P. C., Hsu, T., \& Vanderberg, D. A. 1985, ApJ, 294, 674

Cooke, B. A., Barstow, M. A., Breeveld, E. R., et al. 1992, Nature, 355, 61 Della Ceca, R., Maccacaro, T., Caccianiga, A., et al. 2004, A\&A, 428, 383 Favata, F., Rosner, R., Sciortino, S., \& Vaiana, G. S. 1988, ApJ, 324, 1010 Favata, F., Micela, G., Sciortino, S., \& Vaiana, G. S. 1992, A\&A, 256, 86 Favata, F., Barbera, M., Micela, G., \& Sciortino, S. 1993, A\&A, 277, 428 Feigelson, E. D., \& Montmerle, T. 1999, ARA\&A, 37, 363

Feigelson, E. D., Hornschemeier, A. E., Micela, G., et al. 2004, ApJ, 611, 1107 Gioia, I. M., Maccacaro, T., Schild, R. E., et al. 1990, ApJS, 72, 567

Gorenstein, P., Harnden, F. R., Jr., \& Fabricant, D. G. 1981, I.E.E.E. Trans. Nucl. Sci., NS-28, 869

Guedel, M., Guinan, E. F., \& Skinner, S. L. 1997, ApJ, 483, 947

Guillout, P., Haywood, M., Motch, C., \& Robin, A. C. 1996, A\&A, 316, 89 Haywood, M., Robin, A. C., \& Crézé, M. 1997, A\&A, 320, 440

Henry, J. P., Gioia, I. M., Mullis, C. R., et al. 2001, ApJ, 553, L109

Hoard, D. W., Wachter, S., Lee Clark, L., \& Bowers, T. P. 2002, ApJ, 565, 511

Kastner, J. H., Crigger, L., Rich, M., \& Weintraub, D. 2003, ApJ, 585, 878

Low, F. J., \& Smith, P. S. 2005, ApJ, 631, 1170

Maggio, A., Sciortino, S., Vaiana, G. S., et al. 1987, ApJ, 315, 687

Majer, P., Schmitt, J. H. M. M., Golub, L., et al. 1986, ApJ, 300, 360

Marino, A., Micela, G., Peres, G., \& Sciortino, S. 2003, A\&A, 406, 629

Micela, G. 2003, Astron. Nachr., 324, 77

Micela, G., Sciortino, S., Serio, S., et al. 1985, ApJ, 292, 172

Micela, G., Sciortino, S., Vaiana, G. S., et al. 1988, ApJ, 325, 798

Micela, G., Sciortino, S., \& Favata, F. 1993, ApJ, 412, 618

Micela, G., Sciortino, S., Kashyap, V., Harnden, F. R., \& Rosner, R. 1996, ApJS, 102, 75

Micela, G., Baldi, A., Cocchia, F., et al. 2003, Astron. Nachr., 324, 144

Micela, G., et al. 2006, A\&A, in preparation

Miller, G. E., \& Scalo, J. M. 1979, ApJS, 41, 513

Morrison, R., \& McCammon, D. 1983, ApJ, 270, 119

Robin, A., \& Crézé, M. 1986, A\&A, 157, 71

Ryter, Ch. E. 1996, Ap\&SS, 236, 285

Schmitt, J. H. M. M. 1984, in X-ray Astronomy 84, ed. M. Oda, \& R. Giacconi (Bologna: ISAS), 17

Schmitt, J. H. M. M., Golub, L., Harnden, F. R., et al. 1985, ApJ, 290, 307

Schmitt, J. H. M. M., Collura, A., Sciortino, S., et al. 1990, ApJ, 365, 704

Sciortino, S., Favata, F., \& Micela, G. 1995, A\&A, 296, 370

Sion, E. M., Holberg, J. B., Barstow, M. A., \& Kidder, K. M. 1995, PASP, 107, 232

Smith, R. K., Brickhouse, N. S., Liedahl, D. A., \& Raymond, J. C. 2001, ApJ, 556, L91

Stern, D., Schmitt, J. H. M. M., \& Kahabka, P. T. 1995, ApJ, 448, 683

Tagliaferri, G., Cutispoto, R., Pallavicini, R., Randich, S., \& Pasquini, L. 1994, A\&A, 285, 272

Tovmassian, G. H., Szkody, P., Greiner, J., et al. 2001, A\&A, 379, 199

Twarog, B. A. 1980, ApJ, 242, 242

Vaiana, G. S. 1983, in Solar and Stellar Magnetic Fields: Origins and Coronal Effects, ed. J. O. Stenflo (Dordrecht: Reidel), IAU Symp., 102, 165 Villumsen, J. V. 1985, ApJ, 290, 75

Vuong, M. H., Montmerle, T., Grosso, N., Feigelson, E. D., Verstraete, L., \& Ozawa, H. 2003, A\&A, 408, 581

Wachter, S., Hoard, D. W., Hansen, K. H., et al. 2003, ApJ, 586, 1356

Wielen, R., \& Fuchs, B. 1983, in Kinematics, Dynamics and the Structure of the Milky Way, ed. W. L. H. Shuter (Dordrecht: Reidel), 81

Zuckerman, B., \& Song, I. 2004, ARA\&A, 42, 685 


\section{Online Material}


J. López-Santiago et al.: The stellar content of the XBSS, Online Material p 2

Table 2. Infrared counterparts identified for the stellar sample, with colour information from 2MASS.

\begin{tabular}{|c|c|c|c|c|c|c|c|c|c|}
\hline \multirow[t]{2}{*}{ Id. (XBSS ...) } & \multirow[t]{2}{*}{ Optical counterpart } & \multicolumn{6}{|c|}{ 2MASS counterpart* } & \multicolumn{2}{|c|}{ Spectral type } \\
\hline & & $\begin{array}{c}\text { RA } \\
(\mathrm{J} 2000.0)\end{array}$ & $\begin{array}{c}\text { Dec } \\
(\mathrm{J} 2000.0)\end{array}$ & $\begin{array}{l}\text { Offset } \\
\operatorname{arcsec}\end{array}$ & $\begin{array}{c}\mathrm{J} \\
(\mathrm{mag})\end{array}$ & $\begin{array}{c}\mathrm{H} \\
(\mathrm{mag})\end{array}$ & $\begin{array}{c}\mathrm{K}_{\mathrm{s}} \\
(\mathrm{mag})\end{array}$ & IR count. & Opt. spectrum \\
\hline J001051.6+105140 & - & 00:10:51.39 & $+10: 51: 40.2$ & 3.4 & $12.47 \pm 0.02$ & $11.96 \pm 0.03$ & $11.85 \pm 0.02$ & $\mathrm{~K} 2$ & $\mathrm{~K} 2$ \\
\hline J001749.7+161952 & 39 Psc & $00: 17: 49.97$ & $+16: 19: 51.8$ & 3.5 & $6.29 \pm 0.02$ & $6.09 \pm 0.02$ & $6.05 \pm 0.02$ & F4 & - \\
\hline J002953.1+044524 & - & $00: 29: 53.22$ & $+04: 45: 23.3$ & 1.4 & $10.14 \pm 0.04$ & $9.88 \pm 0.04$ & $9.80 \pm 0.04$ & F6 & - \\
\hline J012757.2+190000 & - & $01: 27: 57.15$ & $+19: 00: 00.9$ & 1.4 & $9.82 \pm 0.02$ & $9.31 \pm 0.03$ & $9.19 \pm 0.02$ & $\mathrm{~K} 2$ & - \\
\hline J012757.3+185923 & $\mathrm{BD}+18193$ & $01: 27: 57.37$ & $+18: 59: 25.0$ & 1.1 & $8.24 \pm 0.02$ & $7.94 \pm 0.03$ & $7.86 \pm 0.02$ & G0 & - \\
\hline J014100.6-675328 & BL Hyi & 01:41:00.38 & $-67: 53: 27.3$ & 2.2 & $14.32 \pm 0.04$ & $13.66 \pm 0.04$ & $13.25 \pm 0.05$ & M6 & $\mathrm{Am}$ \\
\hline J021830.0-045514 & - & 02:18:29.91 & $-04: 55: 13.7$ & 1.6 & $10.92 \pm 0.02$ & $10.28 \pm 0.02$ & $10.06 \pm 0.02$ & M1 & - \\
\hline J040744.6-710846 & - & 04:07:44.64 & $-71: 08: 47.0$ & 0.2 & $14.11 \pm 0.03$ & $13.53 \pm 0.04$ & $13.25 \pm 0.04$ & M4 & M2-3e \\
\hline J040807.2-712702 & EXO 0408.4-7134 & 04:08:07.07 & $-71: 27: 00.9$ & 1.7 & $9.66 \pm 0.02$ & $9.00 \pm 0.02$ & $8.84 \pm 0.03$ & K8 & - \\
\hline J051617.1+794408 & HD 32558 & $05: 16: 17.33$ & $+79: 44: 10.8$ & 2.3 & $9.55 \pm 0.11$ & $9.61 \pm 0.08$ & $9.21 \pm 0.04$ & A7 & - \\
\hline J052155.0-252200 & - & $05: 21: 55.21$ & $-25: 22: 00.7$ & 2.6 & $11.78 \pm 0.03$ & $11.42 \pm 0.02$ & $11.32 \pm 0.02$ & G6 & - \\
\hline J062425.7-642958 & GSC 08902-01379 & $06: 24: 25.81$ & $-64: 29: 57.0$ & 1.4 & $10.21 \pm 0.02$ & $9.89 \pm 0.02$ & $9.80 \pm 0.02$ & G2 & - \\
\hline J074359.7+744057 & - & 07:44:00.60 & $+74: 40: 56.4$ & 3.7 & $11.89 \pm 0.02$ & $11.50 \pm 0.02$ & $11.41 \pm 0.02$ & K0 & K5 \\
\hline J080309.8+650807 & HD 65497 & 08:03:09.50 & $+65: 08: 08.8$ & 2.5 & $6.90 \pm 0.02$ & $6.80 \pm 0.02$ & $6.74 \pm 0.02$ & A8 & - \\
\hline J085427.8+584158 & GSC 03811-00428 & $08: 54: 27.81$ & $+58: 42: 02.0$ & 3.2 & $8.38 \pm 0.02$ & $8.00 \pm 0.04$ & $7.88 \pm 0.02$ & G6 & - \\
\hline J091043.4+054757 & - & 09:10:43.31 & $+05: 48: 01.0$ & 3.6 & $12.82 \pm 0.03$ & $12.29 \pm 0.03$ & $11.95 \pm 0.03$ & M5 & M5 \\
\hline J095955.2+251549 & - & 09:59:55.04 & $+25: 15: 51.0$ & 3.3 & $10.85 \pm 0.02$ & $10.60 \pm 0.02$ & $10.59 \pm 0.02$ & F6 & A9 \\
\hline J102044.1+081424 & - & 10:20:44.06 & $+08: 14: 23.4$ & 1.5 & $10.35 \pm 0.02$ & $9.76 \pm 0.02$ & $9.47 \pm 0.02$ & M4 & M5e \\
\hline J105131.1+573439 & [IUY2001] 2 & $10: 51: 30.93$ & $+57: 34: 39.5$ & 1.5 & $11.04 \pm 0.02$ & $10.47 \pm 0.03$ & $10.21 \pm 0.02$ & M3 & M3-4e \\
\hline $\mathrm{J} 110320.1+355803$ & HD 95735 & 11:03:20.23 & $+35: 58: 11.7$ & 8.2 & $4.20 \pm 0.24$ & $3.64 \pm 0.20$ & $3.25 \pm 0.31$ & M6: & M2 \\
\hline $\mathrm{J} 122655.1+012002$ & - & $12: 26: 54.97$ & $+01: 20: 00.3$ & 3.1 & $14.39 \pm 0.03$ & $13.80 \pm 0.03$ & $13.57 \pm 0.05$ & M2 & M1-3 \\
\hline $\mathrm{J} 122751.2+333842$ & - & $12: 27: 51.19$ & $+33: 38: 43.3$ & 0.9 & $9.44 \pm 0.02$ & $8.82 \pm 0.04$ & $8.64 \pm 0.02$ & M0 & K7 \\
\hline $\mathrm{J} 122837.3+015720$ & GSC 00282-00187 & $12: 28: 37.19$ & $+01: 57: 20.4$ & 2.0 & $10.90 \pm 0.03$ & $10.26 \pm 0.03$ & $10.04 \pm 0.02$ & M2 & - \\
\hline J122942.3+015525 & GSC 00282-00477 & $12: 29: 42.47$ & $+01: 55: 24.8$ & 1.6 & $11.39 \pm 0.02$ & $10.98 \pm 0.02$ & $10.89 \pm 0.03$ & G9 & F9-G2 \\
\hline J123600.7-395217 & CD-39 7717B & $12: 36: 00.55$ & $-39: 52: 15.6$ & 3.1 & $9.15 \pm 0.02$ & $8.53 \pm 0.04$ & $8.35 \pm 0.03$ & K7 & - \\
\hline J124938.7-060444 & IM Vir & $12: 49: 38.68$ & $-06: 04: 44.7$ & 0.7 & $8.20 \pm 0.02$ & $7.74 \pm 0.02$ & $7.67 \pm 0.02$ & K0 & - \\
\hline $\mathrm{J} 133321.2+503102$ & GSC 03469-00261 & $13: 33: 21.00$ & $+50: 31: 03.7$ & 2.9 & $10.17 \pm 0.02$ & $9.90 \pm 0.03$ & $9.88 \pm 0.03$ & F7 & F6: \\
\hline J133626.9-342636 & - & $13: 36: 26.90$ & $-34: 26: 36.5$ & 0.5 & $11.85 \pm 0.04$ & $11.36 \pm 0.04$ & $11.21 \pm 0.03$ & $\mathrm{~K} 2$ & - \\
\hline $\mathrm{J} 134732.0+582103$ & - & 13:47:31.95 & $+58: 21: 03.5$ & 0.5 & $11.24 \pm 0.02$ & $10.60 \pm 0.02$ & $10.41 \pm 0.02$ & K9 & M3e \\
\hline
\end{tabular}


J. López-Santiago et al.: The stellar content of the XBSS, Online Material p 3

Table 2. continued.

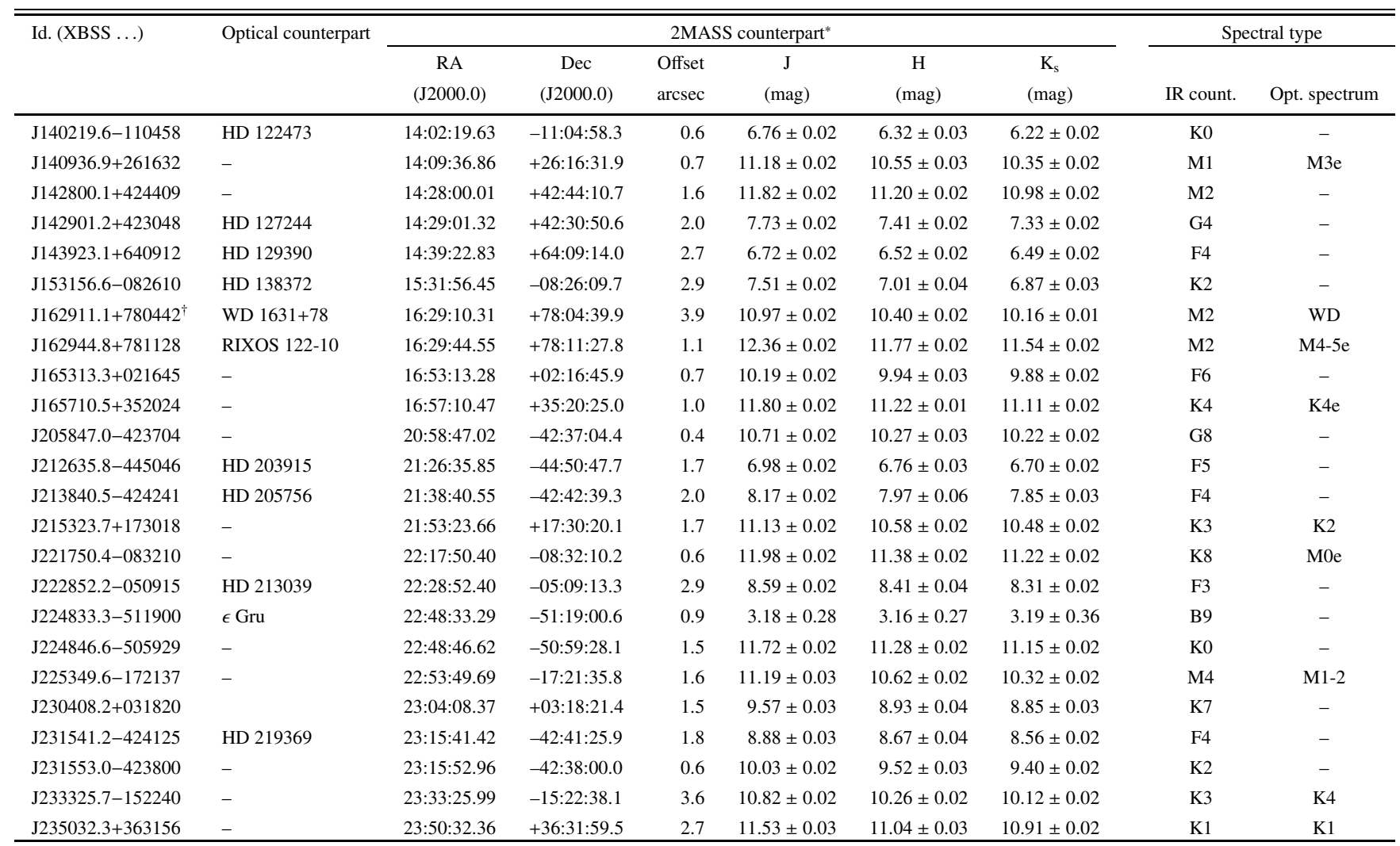

* Errors in the colours refer to the total photometric uncertainty as described in 2MASS.

${ }^{\dagger}$ Binary systems with a white dwarf. 
J. López-Santiago et al.: The stellar content of the XBSS, Online Material $p 4$

Table 3. Best-fit parameters derived from the spectral analysis of the 58 stellar sources in the XMM-Newton Bright Serendipitous survey, with errors measured as the $90 \%$ confidence range.

\begin{tabular}{|c|c|c|c|c|c|c|c|c|}
\hline $\begin{array}{l}\text { Id. } \\
\text { (XBSS ...) }\end{array}$ & $\begin{array}{c}k T_{1} \\
(\mathrm{keV})\end{array}$ & $\begin{array}{c}k T_{2} \\
(\mathrm{keV})\end{array}$ & $E M_{1} / E M_{2}$ & $Z / Z_{\odot}$ & $\begin{array}{c}N_{\mathrm{H}} \\
\left(\times 10^{21} \mathrm{~cm}^{-2}\right)\end{array}$ & $\begin{array}{c}\chi^{2} \\
\text { (d.o.f.) }\end{array}$ & Counts $^{\dagger}$ & $\begin{array}{c}\text { Unabsorbed flux } \\
\left(\times 10^{-13} \mathrm{erg} \mathrm{cm}^{-2} \mathrm{~s}^{-1}\right)\end{array}$ \\
\hline $\mathrm{J} 001002.4+110831$ & $0.98_{-0.11}^{+0.09}$ & - & - & $0.09_{-0.04}^{+0.05}$ & $0.34_{-0.34}^{+0.55}$ & $1.25(66)$ & 828 & $7.31_{-1.01}^{+1.13}$ \\
\hline $\mathrm{J} 001051.6+105140^{* *}$ & $0.10_{-0.02}^{+0.01}$ & $2.01_{-0.33}^{+0.62}$ & 6.60 & $1.33_{-0.36}^{+0.38}$ & $2.12_{-0.40}^{+0.59}$ & $0.69(24)$ & 69 & $0.84_{-0.00}^{+0.00}$ \\
\hline J001749.7+161952 & $0.41_{-0.03}^{+0.04}$ & - & - & $1.09_{-0.08}^{+0.08}$ & $0.00_{-0.00}^{+0.33}$ & $0.85(53)$ & 890 & $1.42_{-1.26}^{+0.14}$ \\
\hline J002953.1+044524 & $0.23_{-0.04}^{+0.04}$ & - & - & $0.94_{-0.12}^{+0.13}$ & $5.89_{-1.01}^{+1.03}$ & $1.13(29)$ & 361 & $9.25_{-8.40}^{+2.38}$ \\
\hline J005822.9-274016 & $0.81_{-0.23}^{+0.19}$ & - & - & $0.04_{-0.03}^{+0.04}$ & $0.13_{-0.13}^{+1.111}$ & $0.81(36)$ & 434 & $1.35_{-0.41}^{+0.32}$ \\
\hline J012757.2+190000 & $1.00_{-0.13}^{+0.10}$ & - & - & $0.14_{-0.07}^{+0.11}$ & $0.00_{-0.00}^{+0.57}$ & $1.13(31)$ & 391 & $5.94_{-2.52}^{+0.15}$ \\
\hline J012757.3+185923 & $0.75_{-0.05}^{+0.04}$ & - & - & $0.16_{-0.05}^{+0.07}$ & $0.25_{-0.25}^{+0.50}$ & $0.94(81)$ & 1086 & $18.73_{-3.18}^{+1.86}$ \\
\hline J014100.6-675328 & $10.19_{-1.08}^{+1.05}$ & - & - & $1.14_{-0.36}^{+0.039}$ & $0.00_{-0.00}^{+0.03}$ & $1.18(328)$ & 9567 & $8.01_{-1.48}^{-2.75}$ \\
\hline J021830.0-045514 & $0.34_{-0.06}^{+0.05}$ & $1.16_{-0.02}^{+0.02}$ & 1.27 & $0.16_{-0.03}^{+0.04}$ & $0.30_{-0.25}^{+0.29}$ & $1.21(226)$ & 6044 & $2.18_{-0.18}^{+0.34}$ \\
\hline J040744.6-710846 & $0.99_{-0.16}^{+0.35}$ & - & - & $0.06_{-0.04}^{+0.07}$ & $0.05_{-0.05}^{+0.05}$ & $1.16(39)$ & 323 & $0.70_{-0.22}^{+0.19}$ \\
\hline J040807.2-712702 & $0.35_{-0.04}^{+0.07}$ & $0.95_{-0.06}^{+0.07}$ & 1.29 & $0.11_{-0.02}^{+0.03}$ & $0.19_{-0.17}^{+0.36}$ & $1.00(215)$ & 3222 & $3.97_{-0.53}^{+0.61}$ \\
\hline J051617.1+794408* & $0.34_{-0.02}^{-0.04}$ & $0.92_{-0.05}^{+0.00}$ & 0.91 & $0.13_{-0.02}^{+0.02}$ & $0.10_{-0.10}^{+0.24}$ & $0.91(332)$ & 5170 & $2.42_{-0.73}^{+0.05}$ \\
\hline J052048.9-454128 & $0.40_{-0.06}^{+0.07}$ & $0.93_{-0.09}^{+0.09}$ & 0.77 & $0.11_{-0.04}^{+0.06}$ & $0.00_{-0.00}^{+0.34}$ & $0.94(263)$ & 3708 & $4.47_{-0.33}^{+0.85}$ \\
\hline J052155.0-252200 & $0.72_{-0.13}^{+0.10}$ & - & - & $0.23_{-0.14}^{+0.70}$ & $3.37_{-1.16}^{+1.22}$ & $0.75(54)$ & 220 & $1.31_{-0.99}^{+0.27}$ \\
\hline J062425.7-642958 & $0.47_{-0.19}^{+0.11}$ & - & - & $0.11_{-0.08}^{+0.54}$ & $1.34_{-1.34}^{+3.21}$ & $0.66(35)$ & 227 & $1.73_{-1.14}^{+0.37}$ \\
\hline J074359.7+744057 & $0.30_{-0.04}^{-0.19}$ & $1.20_{-0.20}^{+0.40}$ & 5.43 & $0.08_{-0.06}^{+0.08}$ & $2.73_{-1.09}^{+1.06}$ & $0.92(65)$ & 1430 & $4.03_{-3.53}^{+0.014}$ \\
\hline J080309.8+650807 & $0.50_{-0.03}^{+0.05}$ & - & - & $1.45_{-0.14}^{+0.71}$ & $0.00_{-0.00}^{+0.80}$ & $1.40(59)$ & 707 & $0.96_{-0.48}^{+0.13}$ \\
\hline J085427.8+584158 & $0.61_{-0.08}^{+0.06}$ & - & - & $0.09_{-0.04}^{+0.06}$ & $0.00_{-0.00}^{+0.52}$ & $0.71(62)$ & 945 & $1.36_{-0.35}^{+0.21}$ \\
\hline J091043.4+054757 & $1.02_{-0.22}^{+0.26}$ & - & - & $0.04_{-0.03}^{+0.05}$ & $0.21_{-0.21}^{+0.73}$ & $1.05(74)$ & 1235 & $1.18_{-0.23}^{+0.26}$ \\
\hline J095955.2+251549 & $1.01_{-0.06}^{-0.22}$ & - & - & $0.12_{-0.03}^{+0.04}$ & $0.76_{-0.35}^{+0.21}$ & 1.05 (114) & 3214 & $1.39_{-0.23}^{+0.23}$ \\
\hline $\mathrm{J} 102044.1+081424$ & $0.33_{-0.03}^{+0.04}$ & - & - & $0.03_{-0.02}^{+0.03}$ & $1.33_{-0.46}^{+0.45}$ & $1.15(48)$ & 1117 & $2.72_{-1.58}^{+0.34}$ \\
\hline J105131.1+573439 & $0.32_{-0.08}^{+0.08}$ & $0.93_{-0.14}^{+0.18}$ & 1.14 & $0.15_{-0.06}^{+0.20}$ & $0.05_{-0.05}^{+0.90}$ & $0.94(79)$ & 1834 & $1.02_{-0.53}^{+0.10}$ \\
\hline $\mathrm{J} 110320.1+355803$ & $0.27_{-0.02}^{+0.02}$ & $0.71_{-0.06}^{+0.13}$ & 4.18 & $0.06_{-0.01}^{+0.001}$ & $0.00_{-0.00}^{+0.01}$ & $1.29(391)$ & 11147 & $3.97_{-2.03}^{+1.92}$ \\
\hline $\mathrm{J} 122751.2+333842$ & $0.26_{-0.03}^{-0.02}$ & $0.89_{-0.08}^{+0.00}$ & 1.23 & $0.21_{-0.09}^{+0.26}$ & $0.00_{-0.00}^{+0.71}$ & $0.70(67)$ & 1844 & $1.01_{-0.77}^{+0.03}$ \\
\hline $\mathrm{J} 122655.1+012002$ & $1.08_{-0.31}^{+0.31}$ & - & - & $0.08_{-0.07}^{+0.16}$ & $0.00_{-0.00}^{+0.11}$ & $0.80(59)$ & 708 & $0.33_{-0.26}^{+0.09}$ \\
\hline $\mathrm{J} 122837.3+015720$ & $0.27_{-0.04}^{-0.01}$ & $1.04_{-0.08}^{+0.10}$ & 1.99 & $0.17_{-0.06}^{+0.08}$ & $1.12_{-0.74}^{+1.30}$ & $0.81(135)$ & 1857 & $1.78_{-0.83}^{+0.34}$ \\
\hline J122942.3+015525 & $0.29_{-0.06}^{+0.07}$ & $1.03_{-0.10}^{+0.13}$ & 0.90 & $0.35_{-0.14}^{+0.27}$ & $1.94_{-1.13}^{+1.120}$ & $0.96(79)$ & 1059 & $1.72_{-0.78}^{+0.33}$ \\
\hline $\mathrm{J} 123208.7+640304$ & $0.25_{-0.07}^{+0.10}$ & $1.09_{-0.15}^{+0.23}$ & 1.09 & $0.10_{-0.07}^{+0.24}$ & $0.28_{-0.28}^{+1.95}$ & $0.85(44)$ & 534 & $0.64_{-0.40}^{+0.14}$ \\
\hline J123549.1-395026 & $0.32_{-0.01}^{+0.01}$ & $0.90_{-0.04}^{+0.04}$ & 1.73 & $0.19_{-0.04}^{+0.04}$ & $0.00_{-0.00}^{+0.27}$ & $1.08(216)$ & 6004 & $2.16_{-0.58}^{+0.19}$ \\
\hline $\mathrm{J} 123600.7-395217^{*}$ & $0.31_{-0.01}^{+0.01}$ & $1.01_{-0.03}^{+0.02}$ & 0.81 & $0.22_{-0.04}^{+0.04}$ & $0.00_{-0.00}^{+0.01}$ & $1.42(617)$ & 34052 & $14.05_{-3.64}^{+2.45}$ \\
\hline J124938.7-060444 & $0.64_{-0.01}^{+0.01}$ & $1.21_{-0.02}^{+0.02}$ & 0.83 & $0.17_{-0.01}^{+0.01}$ & $0.01_{-0.01}^{+0.02}$ & $1.15(517)$ & 40463 & $22.39_{-1.46}^{+0.66}$ \\
\hline $\mathrm{J} 133321.2+503102$ & $0.60_{-0.04}^{+0.04}$ & - & - & $0.09_{-0.02}^{+0.01}$ & $0.98_{-0.38}^{+0.41}$ & $1.09(170)$ & 1190 & $1.25_{-0.20}^{+0.24}$ \\
\hline J133626.9-342636 & $0.98_{-0.41}^{+0.32}$ & - & - & $0.07_{-0.06}^{+0.15}$ & $0.03_{-0.03}^{+2.15}$ & $0.48(16)$ & 466 & $0.51_{-0.35}^{+0.15}$ \\
\hline $\mathrm{J} 134732.0+582103$ & $0.82_{-0.03}^{-0.03}$ & - & - & $0.06_{-0.01}^{+0.001}$ & $0.00_{-0.00}^{+0.15}$ & $1.06(177)$ & 4120 & $1.75_{-0.16}^{+0.15}$ \\
\hline J140219.6-110458 & $0.44_{-0.12}^{-0.03}$ & - & - & $1.11_{-0.94}^{+3.01}$ & $2.76_{-1.68}^{+1.03}$ & $1.02(39)$ & 491 & $1.16_{-0.62}^{+0.10}$ \\
\hline $\mathrm{J} 140936.9+261632$ & $0.35_{-0.06}^{+0.09}$ & $0.94_{-0.18}^{+0.17}$ & 2.48 & $0.08_{-0.08}^{+0.06}$ & $0.29_{-0.29}^{+1.03}$ & $1.04(95)$ & 2402 & $0.78_{-0.29}^{+0.13}$ \\
\hline $\mathrm{J} 142800.1+424409$ & $0.28_{-0.05}^{+0.08}$ & $0.93_{-0.11}^{+0.12}$ & 1.87 & $0.23_{-0.11}^{+0.36}$ & $1.00_{-1.00}^{+2.07}$ & $0.84(90)$ & 1226 & $1.07_{-0.40}^{+0.18}$ \\
\hline J142901.2+423048 & $0.49_{-0.08}^{+0.06}$ & - & - & $0.99_{-0.72}^{+4.00}$ & $0.00_{-0.00}^{+0.42}$ & $1.15(59)$ & 805 & $0.70_{-0.66}^{+0.50}$ \\
\hline J143923.1+640912 & $0.49_{-0.03}^{+0.02}$ & - & - & $1.11_{-0.62}^{+3.89}$ & $0.01_{-0.01}^{+0.09}$ & $1.35(120)$ & 2155 & $1.61_{-1.35}^{+0.26}$ \\
\hline $\mathrm{J} 153156.6-082610$ & $0.57_{-0.32}^{+0.08}$ & - & - & $0.11_{-0.04}^{+0.22}$ & $1.07_{-0.98}^{+3.43}$ & $0.87(84)$ & 909 & $0.88_{-0.82}^{+1.63}$ \\
\hline $\mathrm{J} 162911.1+780442$ & $0.70_{-0.41}^{+0.20}$ & - & - & $0.04_{-0.02}^{+0.02}$ & $0.00_{-0.00}^{+0.92}$ & $1.00(21)$ & 280 & $1.13_{-0.88}^{+0.08}$ \\
\hline $\mathrm{J} 162944.8+781128$ & $0.74_{-0.23}^{+0.16}$ & - & - & $0.10_{-0.08}^{+0.29}$ & $0.10_{-0.10}^{+2.27}$ & $1.09(9)$ & 150 & $0.80_{-0.78}^{+0.19}$ \\
\hline $\mathrm{J} 165313.3+021645$ & $0.64_{-0.05}^{+0.04}$ & - & - & $0.56_{-0.30}^{+0.40}$ & $0.09_{-0.09}^{+1.118}$ & $0.93(79)$ & 1318 & $0.70_{-0.67}^{+0.15}$ \\
\hline $\mathrm{J} 165710.5+352024^{* *}$ & $0.85_{-0.36}^{+0.28}$ & - & - & $0.08_{-0.07}^{+0.33}$ & $0.06_{-0.06}^{+3.11}$ & $1.21(7)$ & 232 & $1.24_{-0.60}^{+0.35}$ \\
\hline J205847.0-423704 & $0.74_{-0.09}^{+0.06}$ & - & - & $0.14_{-0.06}^{+0.07}$ & $0.34_{-0.34}^{+0.57}$ & $1.21(72)$ & 1096 & $0.71_{-0.31}^{+0.18}$ \\
\hline J212635.8-445046 & $0.62_{-0.06}^{+0.05}$ & - & - & $0.11_{-0.04}^{+0.07}$ & $0.17_{-0.17}^{+0.78}$ & $1.14(78)$ & 1899 & $1.52_{-0.70}^{+0.31}$ \\
\hline $\mathrm{J} 213840.5-424241$ & $0.47_{-0.14}^{-0.09}$ & - & - & $0.97_{-0.13}^{+0.13}$ & $0.00_{-0.00}^{+1.193}$ & $0.97(118)$ & 1261 & $0.72_{-0.67}^{+0.06}$ \\
\hline $\mathrm{J} 215323.7+173018$ & $1.31_{-0.29}^{-0.48}$ & - & - & $0.14_{-0.11}^{+0.30}$ & $1.05_{-1.05}^{+1.136}$ & $0.98(75)$ & 507 & $0.69_{-0.21}^{+0.27}$ \\
\hline
\end{tabular}


J. López-Santiago et al.: The stellar content of the XBSS, Online Material p 5

Table 3. continued.

\begin{tabular}{|c|c|c|c|c|c|c|c|c|}
\hline $\begin{array}{l}\text { Id. } \\
\text { (XBSS ...) }\end{array}$ & $\begin{array}{c}k T_{1} \\
(\mathrm{keV})\end{array}$ & $\begin{array}{c}k T_{2} \\
(\mathrm{keV})\end{array}$ & $E M_{1} / E M_{2}$ & $Z / Z_{\odot}$ & $\begin{array}{c}N_{\mathrm{H}} \\
\left(\times 10^{21} \mathrm{~cm}^{-2}\right)\end{array}$ & $\begin{array}{c}\chi^{2} \\
\text { (d.o.f.) }\end{array}$ & Counts $^{\dagger}$ & $\begin{array}{c}\text { Unabsorbed flux } \\
\left(\times 10^{-13} \mathrm{erg} \mathrm{cm}^{-2} \mathrm{~s}^{-1}\right)\end{array}$ \\
\hline $\mathrm{J} 221750.4-083210$ & $0.23_{-0.04}^{+0.05}$ & $1.09_{-0.22}^{+0.28}$ & 3.61 & $0.71_{-0.12}^{+0.11}$ & $2.44_{-1.59}^{+1.15}$ & $0.99(49)$ & 546 & $1.76_{-1.43}^{+0.09}$ \\
\hline $\mathrm{J} 222852.2-050915$ & $0.52_{-0.07}^{+0.05}$ & - & - & $0.50_{-0.23}^{+1.92}$ & $0.36_{-0.36}^{+0.88}$ & $1.04(131)$ & 1550 & $0.92_{-0.62}^{+0.18}$ \\
\hline $\mathrm{J} 224833.3-511900$ & $0.30_{-0.03}^{+0.03}$ & - & - & $0.65_{-0.14}^{+0.13}$ & $4.94_{-0.52}^{+0.73}$ & $1.03(26)$ & 170 & $2.41_{-2.26}^{+0.64}$ \\
\hline J224846.6-505929 & $0.69_{-0.48}^{+0.14}$ & - & - & $0.09_{-0.07}^{+0.53}$ & $0.00_{-0.00}^{+2.48}$ & $0.55(20)$ & 139 & $0.52_{-0.39}^{+0.16}$ \\
\hline $\mathbf{J} 225349.6-172137^{* *}$ & $0.13_{-0.06}^{+0.05}$ & - & - & $4.74_{-4.72}^{+0.26}$ & $8.88_{-3.85}^{+8.46}$ & $0.94(20)$ & 71 & $61.89_{-61.85}^{+2.00}$ \\
\hline $\mathrm{J} 230408.2+031820$ & $0.31_{-0.05}^{+0.09}$ & $0.91_{-0.09}^{+0.13}$ & 1.48 & $0.15_{-0.05}^{+0.16}$ & $0.54_{-0.12}^{+0.17}$ & $1.23(81)$ & 1936 & $4.17_{-0.90}^{+0.69}$ \\
\hline $\mathrm{J} 231541.2-424125$ & $0.47_{-0.07}^{+0.07}$ & - & - & $0.71_{-0.53}^{+4.29}$ & $0.19_{-0.19}^{+0.89}$ & $1.08(79)$ & 862 & $0.69_{-0.63}^{+0.17}$ \\
\hline $\mathrm{J} 231553.0-423800$ & $0.78_{-0.17}^{+0.26}$ & - & - & $0.02_{-0.01}^{+0.02}$ & $0.92_{-0.80}^{+0.79}$ & $1.01(70)$ & 1247 & $0.73_{-0.21}^{+0.16}$ \\
\hline J233325.7-152240 & $0.29_{-0.07}^{+0.08}$ & $0.97_{-0.02}^{+0.03}$ & 2.41 & $0.09_{-0.02}^{+0.05}$ & $1.09_{-0.60}^{+0.54}$ & $1.31(153)$ & 2853 & $2.35_{-0.91}^{+0.33}$ \\
\hline $\mathrm{J} 235032.3+363156$ & $1.13_{-0.14}^{+0.14}$ & - & - & $0.34_{-0.10}^{+0.09}$ & $0.00_{-0.00}^{+0.52}$ & $0.77(29)$ & 152 & $1.05_{-0.64}^{+0.24}$ \\
\hline
\end{tabular}

$\dagger$ Total net counts in the EPIC (MOS + PN) chips.

* Unabsorbed flux in $0.5-10 \mathrm{keV}$.

* For these sources, a 3T-model has been used to obtain an accurate fitting to the hard tail of their X-ray spectrum. The parameters of their third thermal component are a) XBSS J051617.1+794408 (HD 32558): $k T_{3}=4.32_{-2.50}^{+3.28} \mathrm{keV}, E M_{1} / E M_{3}=3.57$ and b) XBSS J123600.7-395217 (CD-39 7717B, TWA 11B): $k T_{3}=2.39_{-0.15}^{+0.16} \mathrm{keV}, E M_{1} / E M_{3}=0.75$.

** Problematic sources. The fitting in these stars is much less accurate due to the low number of counts in their spectra after background subtraction. 